\title{
Measure rigidity of synthetic lower Ricci curvature bound on Riemannian manifolds
}

\author{
Bang-Xian Han*
}

August 17, 2021

\begin{abstract}
In this paper we investigate Lott-Sturm-Villani's synthetic lower Ricci curvature bound on Riemannian manifolds with boundary. We prove several measure rigidity results related to optimal transport on Riemannian manifolds, which completely characterize $\operatorname{CD}(K, \infty)$ condition and non-collapsed $\mathrm{CD}(K, N)$ condition on Riemannian manifolds with boundary. In particular, we reveal the measure rigidity of Riemannian interpolation inequality proved by Cordero-Erausquin, McCann and Schmuckenschläger. We prove that log(semi)concave measures are the only reference measures so that displacement convexity holds on Riemannian manifolds. This is the first measure rigidity result concerning the synthetic dimension-free $\mathrm{CD}$ condition, which is new even on $\mathbb{R}^{n}$. Using $L^{1}$-optimal transportation theory, we also prove that $\mathrm{CD}$ condition yields geodesical convexity (with respect to the usual Riemannian distance).
\end{abstract}

Keywords: measure rigidity, curvature-dimension condition, metric measure space, Riemannian manifold, boundary, Bakry-Émery theory, optimal transport.

\section{Contents}

1 Introduction 2

2 Smooth metric measure spaces with boundary $\quad 7$

3 Main results: measure rigidity theorems 10

3.1 Measure rigidity: absolute continuity . . . . . . . . . . . . . 11

3.2 Measure rigidity: regularity of density, non-collapsing . . . . . . . . . 17

3.3 Measure rigidity: geodesical convexity . . . . . . . . . . . . . . 23

*Department of Mathematics, Technion-Israel Institute of Technology, han@technion.ac.il 


\section{Introduction}

The synthetic theory of metric measure spaces with lower Ricci curvature bounds, initiated by Lott-Villani [25] and Sturm [30,31], has remarkable developments in recent years. We refer the reader to the survey [3] for an overview of this topic and bibliography.

Many important results, previously known on Riemannian manifolds with lower Ricci curvature bound, now have their generalized versions in synthetic setting. However, we still do not fully understand synthetic lower Ricci curvature bound on Riemannian manifolds. In particular, we know very little about the dimension-free $\mathrm{CD}(K, \infty)$ condition.

In this paper we return to the starting point of this rapidly developing theory, investigate the following conjecture using new tools and results developed in recent years.

Conjecture: Let $(M, \mathrm{~g})$ be a complete Riemannian manifold and $\Omega$ be a bounded open set. Denote by $\mathrm{d}_{\Omega}$ the intrinsic distance on $\bar{\Omega}$ induced by $\mathrm{g}$. Then $\left(\bar{\Omega}, \mathrm{d}_{\Omega}, \mathfrak{m}\right)$ is $\mathrm{CD}(K, \infty)$ in the sense of Lott-Sturm-Villani if and only if the following conditions are satisfied:

1) $\mathfrak{m}=e^{-V}$ Vol for some semi-convex function $V$,

2) $\Omega$ is geodesically convex.

Next, we introduce some backgrounds and explain the motivation in more detail. Let $(M, \mathrm{~g})$ be a $n$-dimensional Riemannian manifold, and $\mathfrak{m}:=e^{-V} \operatorname{Vol}_{\mathrm{g}}$ be a weighted measure for some smooth function $V$. The diffusion operator associated with the smooth metric measure space $(M, \mathrm{~g}, \mathfrak{m})$ is $\mathrm{L}=\Delta-\nabla V$ where $\Delta$ is the Laplace-Beltrami operator, and the well-known Bakry-Émery's $\Gamma_{2}$ operator is defined by

$$
\Gamma_{2}(f):=\frac{1}{2} \mathrm{~L} \Gamma(f, f)-\Gamma(f, \mathrm{~L} f), \quad \Gamma(f, f):=\frac{1}{2} \mathrm{~L}\left(f^{2}\right)-f \mathrm{~L} f .
$$

It is known that $\Gamma(\cdot, \cdot)=\mathrm{g}(\nabla \cdot, \nabla \cdot)$, and we have the following generalized Bochner's formula

$$
\Gamma_{2}(f)=\operatorname{Ricci}(\nabla f, \nabla f)+\operatorname{Hess}_{V}(\nabla f, \nabla f)+\left\|\operatorname{Hess}_{f}\right\|_{\mathrm{HS}}^{2}
$$

for any $f \in C^{\infty}(M)$, where $\operatorname{Hess}_{V}=\mathrm{D}^{2} V$ is the Hessian of $V$ and $\left\|\operatorname{Hess}_{f}\right\|_{\mathrm{HS}}$ is the Hilbert-Schmidt norm of $\operatorname{Hess}_{f}$. We say that $(M, \mathrm{~g}, \mathfrak{m})$ satisfies the $(K, N)$-BakryÉmery's condition (or $\mathrm{BE}(K, N)$ condition for short), if the following generalized Bochner inequality holds

$$
\Gamma_{2}(f) \geq K \Gamma(f)+\frac{1}{N}(\mathrm{~L} f)^{2}, \quad \forall f \in C^{\infty}(M) .
$$

It is known that $\mathrm{BE}(K, N)$ condition yields many important geometric and analytic properties. For example, when $N=\infty$, we have the following equivalent characterizations, which are also regarded as generalized lower Ricci curvature bound (c.f. [33]). 
0) Modified Ricci tensor bound:

$$
\operatorname{Ricci}_{V}(\nabla f, \nabla f):=\operatorname{Ricci}(\nabla f, \nabla f)+\operatorname{Hess}_{V}(\nabla f, \nabla f) \geq K|\nabla f|^{2}
$$

for all $f \in C^{\infty}(M)$.

1) $\operatorname{BE}(K, \infty)$ condition: $\Gamma_{2}(f) \geq K \Gamma(f)$ for all $f \in C^{\infty}(M)$.

2) $\mathrm{CD}(K, \infty)$ condition: $K$-displacement convexity of the entropy functional $\operatorname{Ent}(\cdot \mid \mathfrak{m})$ on $L^{2}$-Wasserstein space $\mathcal{W}_{2}(M)=\left(\mathcal{P}_{2}(M), W_{2}\right)$ (with respect to the Riemannian distance $\left.\mathrm{d}_{\mathrm{g}}\right)$. This means, for any $\mu_{0}, \mu_{1} \in \mathcal{P}_{2}(M)$ with $\mu_{0}, \mu_{1} \ll \mathfrak{m}$, there is a $L^{2}$-Wasserstein geodesic $\left(\mu_{t}\right)_{t \in[0,1]}$ such that

$$
\frac{K}{2} t(1-t) W_{2}^{2}\left(\mu_{0}, \mu_{1}\right)+\operatorname{Ent}\left(\mu_{t} \mid \mathfrak{m}\right) \leq t \operatorname{Ent}\left(\mu_{1} \mid \mathfrak{m}\right)+(1-t) \operatorname{Ent}\left(\mu_{0} \mid \mathfrak{m}\right)
$$

where $\operatorname{Ent}\left(\mu_{t} \mid \mathfrak{m}\right):=\int \ln \rho_{t} \mathrm{~d} \mu_{t}$ if $\mu_{t}=\rho_{t} \mathfrak{m}$.

3) Gradient estimate of heat semi-group:

$$
\left|\nabla \mathrm{H}_{t}(f)\right|^{2} \leq e^{-2 K t} \mathrm{H}_{t}\left(|\nabla f|^{2}\right)
$$

for any $f \in W^{1,2}(M, \mathfrak{m})$, where $\left(\mathrm{H}_{t}\right)_{t>0}$ is the semi-group generated by the diffusion operator L.

Let $\Omega \subset M$ be an open set with smooth boundary, and $\mathrm{d}_{\Omega}$ be (the completion of) the intrinsic distance on $\bar{\Omega}$ induced by the Riemannian distance $\mathrm{d}_{\mathrm{g}}$. Concerning the metric measure space $\left(\bar{\Omega}, \mathrm{d}_{\Omega}, e^{-V} \mathrm{Vol}_{\mathrm{g}}\right)$, one would ask the following questions.

Q-1 What is Bakry-Émery's $\Gamma$-calculus on $\left(\bar{\Omega}, \mathrm{d}_{\Omega}, e^{-V} \operatorname{Vol}_{\mathrm{g}}\right)$, and what does $\Gamma_{2} \geq$ $K \Gamma$ mean in this case?

Q-2 What does $\operatorname{CD}(K, \infty)$ condition (1.3) imply? Can we say that $\Omega$ is geodesically convex?

Q-3 Does the gradient estimate (1.4) of (Neumann) heat semi-group imply geodesical convexity?

Firstly, in Section 2 we study the Bakry-Émery's $\Gamma$-calculus on smooth metric measure spaces with smooth boundary. Using the vocabularies and results developed in non-smooth theory (c.f. [29] and [17]), we define a measure-valued Ricci tensor $\operatorname{Ricci}_{\Omega}$ by

$$
\operatorname{Ricci}_{\Omega}(\cdot, \cdot)=\operatorname{Ricci}_{V}(\cdot, \cdot) e^{-V} \operatorname{Vol}_{\mathrm{g}}+\left.I I(\cdot, \cdot) e^{-V} \mathcal{H}^{n-1}\right|_{\partial \Omega}
$$

where Ricci $_{V}=$ Ricci + Hess $_{V}$ is the Bakry-Émery's modified Ricci tensor and II is the second fundamental form. Combining with the results in $[7,8]$ and [17], we can see that the measure-valued Bochner inequality $\mathbf{R i c c i}_{\Omega} \geq K$ is equivalent to the non-smooth $\operatorname{BE}(K, \infty)$ condition and the Lott-Sturm-Villani's $\mathrm{CD}(K, \infty)$ condition. More precisely, recall that a connected Riemannian manifold with boundary is geodesically convex if and only if $I I \geq 0$ (c.f. Theorem 1.2.1 [34]), we have the following theorem. 
Theorem 1.1 (Measure-valued Ricci tensor, Theorem 2.4 and Corollary 2.5). Let $\left(M, \mathrm{~g}, e^{-V} \mathrm{Vol}_{\mathrm{g}}\right)$ be a n-dimensional weighted Riemannian manifold and $\Omega \subset M$ be a submanifold with $(n-1)$-dimensional smooth orientable boundary. Then Gigli's measure-valued Ricci tensor (c.f. [17]) on $\left(\bar{\Omega}, \mathrm{d}_{\Omega}, e^{-V} \mathrm{Vol}_{\mathrm{g}}\right)$ is given by

$$
\operatorname{Ricci}_{\Omega}(\nabla g, \nabla g)=\operatorname{Ricci}_{V}(\nabla g, \nabla g) e^{-V} \operatorname{Vol}_{g}+\left.I I(\nabla g, \nabla g) e^{-V} \mathcal{H}^{n-1}\right|_{\partial \Omega}
$$

for any $g \in C_{c}^{\infty}$ with $\mathrm{g}(\mathrm{N}, \nabla g)=0$, where $\mathrm{N}$ is the outward normal vector field on $\partial \Omega$.

Furthermore, $\left(\bar{\Omega}, \mathrm{d}_{\Omega}, e^{-V} \operatorname{Vol}_{\mathrm{g}}\right)$ is a $\mathrm{CD}(K, \infty)$ space if and only if $\mathrm{Ricci}_{V} \geq K$, $I I \geq 0$ and path-connected.

On the other side, from $[6,7]$ we know that $\left(\bar{\Omega}, \mathrm{d}_{\Omega}, e^{-V} \operatorname{Vol}_{\mathrm{g}}\right)$ is $\operatorname{CD}(K, \infty)$ if and only if the gradient estimate (1.4) holds. It is proved by F.-Y. Wang (c.f. Chapter 3 in [34]), that the gradient estimate (1.4) is equivalent to Ricci $\geq K$ and $I I \geq 0$. Thus we completely answer the questions Q-1, Q-2 and Q-3.

In the discussions above, we have assumed that $\partial \Omega$ is smooth and there is no mass on the boundary. However, none of these assumptions is necessary or natural in abstract metric measure setting. Precisely, for any $\Omega$ and any Borel measure $\mathfrak{m}$ with full support. It is always meaningful to investigate the $K$-displacement convexity of $\operatorname{Ent}(\cdot \mid \mathfrak{m})$ on $L^{2}$-Wasserstein space $\mathcal{W}_{2}\left(\bar{\Omega}, \mathrm{d}_{\Omega}\right)$, which is exactly the approach used by Lott-Sturm-Villani to define synthetic lower Ricci curvature bound (i.e. $\mathrm{CD}(K, \infty)$ conditions, c.f. $[25,30,31])$.

Let $\left(e_{t}\right)_{t \in[0,1]}$ denote the evaluation maps on a metric space $(X, \mathrm{~d})$ :

$$
e_{t}: \operatorname{Geo}(\mathrm{X}, \mathrm{d}) \ni \gamma \mapsto \gamma_{\mathrm{t}} \in \mathrm{X} .
$$

A measure $\Pi \in \mathcal{P}(\operatorname{Geo}(\mathrm{X}, \mathrm{d}))$ is called an optimal dynamical plan if $\left(e_{0}, e_{1}\right)_{\sharp} \Pi$ is an optimal transportation plan; it easily follows that $[0,1] \ni t \mapsto\left(e_{t}\right)_{\sharp} \Pi$ is a geodesic in $\mathcal{W}_{2}(X, \mathrm{~d})$. Conversely, it is known that any geodesic $\left(\mu_{t}\right)_{t \in[0,1]}$ in $\mathcal{W}_{2}(X, \mathrm{~d})$ can be lifted to an optimal dynamical plan $\Pi$ so that $\left(e_{t}\right)_{\sharp} \Pi=\mu_{t}$ for all $t \in[0,1]$ (c.f. [4, Theorem 2.10]).

Now we are ready to introduce the following $\mathrm{CD}(K, N)$ condition.

Definition 1.2 (Definition 1.3 [31]). Let $K \in \mathbb{R}$ and $N \in[1, \infty]$. We say that $\left(\bar{\Omega}, \mathrm{d}_{\Omega}, \mathfrak{m}\right)$ is a $\operatorname{CD}(K, N)$ space if for any pair $\mu_{0}, \mu_{1} \in \mathcal{P}_{2}(\bar{\Omega})$ with $\mu_{0}, \mu_{1} \ll \mathfrak{m}$, there exists a $L^{2}$-Wasserstein geodesic $\left(\mu_{t}\right)_{t \in[0,1]}$ connecting $\mu_{0}, \mu_{1}$ such that

$$
\mathrm{S}_{N}\left(\mu_{t} \mid \mathfrak{m}\right) \leq-\int\left[\tau_{K, N}^{(t)}\left(\mathrm{d}_{\Omega}\left(\gamma_{0}, \gamma_{1}\right)\right) \rho_{1}^{-\frac{1}{N}}\left(\gamma_{1}\right)+\tau_{K, N}^{(1-t)}\left(\mathrm{d}_{\Omega}\left(\gamma_{0}, \gamma_{1}\right)\right) \rho_{0}^{-\frac{1}{N}}\left(\gamma_{0}\right)\right] \mathrm{d} \Pi(\gamma)
$$

for all $t \in[0,1]$ and some distortion coefficients $\tau_{K, N}^{(t)}$, where $\Pi \in \mathcal{P}\left(\operatorname{Geo}\left(\bar{\Omega}, \mathrm{d}_{\Omega}\right)\right)$ is a lifting of $\left(\mu_{t}\right)$ satisfying $\left(e_{t}\right)_{\sharp} \Pi=\mu_{t}, \mathrm{~S}_{N}\left(\mu_{t} \mid \mathfrak{m}\right)=-\int \rho_{t}^{-\frac{1}{N}} \mathrm{~d} \mu_{t}$ and $\mu_{t}=\rho_{t} \mathfrak{m}$.

In addition, the heat semi-group on $\left(\bar{\Omega}, \mathrm{d}_{\Omega}, \mathfrak{m}\right)$ can be defined as the $L^{2}$-gradient flow of the energy form $\mathcal{E}(f): W^{1,2}(\bar{\Omega}, \mathfrak{m}) \ni f \mapsto \int|\nabla f|^{2} \mathrm{~d} \mathfrak{m}$. So gradient estimate (1.4) is also well-posed for general $\Omega$ and $\mathfrak{m}$. 
In $[7,8]$, Ambrosio-Gigli-Savaré prove that a $\mathrm{CD}(K, \infty)$ metric measure space whose Sobolev space $W^{1,2}$ is Hilbert (a notion named infinitesimally Hilbertianity in the subsequent [16]) is characterised by the validity of the gradient estimate (1.4).

Therefore we have the following natural questions.

Q-4 If the boundary of $\Omega$ is not smooth or $\left.\mathfrak{m}\right|_{\partial \Omega} \neq 0$, such that $\left(\bar{\Omega}, \mathrm{d}_{\Omega}, \mathfrak{m}\right)$ is $\mathrm{CD}(K, \infty)$, can we say that $\Omega$ is geodesically convex as in Theorem 1.1?

Q-5 If there exists a measure $\mathfrak{m}$ with full support such that $\left(\bar{\Omega}, \mathrm{d}_{\Omega}, \mathfrak{m}\right)$ is $\operatorname{CD}(K, \infty)$ (or $\operatorname{CD}(K, N))$. Can we assert $\mathfrak{m} \ll \operatorname{Vol}_{\mathrm{g}}$ ? Is $\left(\bar{\Omega}, \mathrm{d}_{\Omega}, \mathfrak{m}\right)$ a $\operatorname{RCD}(K, \infty)$ space?

In Section 3 we answer these questions completely. It should be noticed that Riemannian manifolds with boundary are usually non-smooth metric measure spaces, even if the boundary is smooth. In the presence of an obstacle, the behavior of the geodesics is quite involved. For example, the regularity of the geodesics can not be better than $C^{1}$ in general (c.f. [1] and [2]). In addition, in many problems related to regularity, (local) convexity plays essential roles. Therefore it is difficult to solve this problem with classical analysis and (second order) PDE methods. However, using optimal transportation theory as an effective tool, it will not be more difficult to study non-smooth boundaries than smooth ones.

Firstly we show the absolute continuity of the reference measure and the regularity of its density. The following theorem improves the results proved by CavallettiMondino [10] and Kell [23] for essentially non-branching $\operatorname{MCP}(K, N)$ spaces. To the knowledge of the author, this is the first measure rigidity result without dimension bound.

Theorem 1.3 (Measure rigidity: absolute continuity and regularity, Proposition 3.1 3.3 3.5). Let $\left(M, \mathrm{~g}, \mathrm{Vol}_{\mathrm{g}}\right)$ be a complete $n$-dimensional Riemannian manifold without boundary, $\mathrm{d}_{\mathrm{g}}$ be the Riemannian distance induced by $\mathrm{g}$ and $\mathfrak{m}$ be a Borel measure with full support on $M$. Then we have the following results.

1) Assume $\left(M, \mathrm{~d}_{\mathrm{g}}, \mathfrak{m}\right)$ satisfies the $\mathrm{CD}(K, \infty)$ condition for some $K \in \mathbb{R}$. Then there is a locally Lipschitz semi-convex functions $V$ on $M$ such that $\mathfrak{m}=$ $e^{-V} \mathrm{Vol}_{\mathrm{g}}$.

2) Assume $\left(M, \mathrm{~d}_{\mathrm{g}}, \mathfrak{m}\right)$ satisfies the $\operatorname{MCP}(K, N)$ condition for some $K \in \mathbb{R}$ and $N<\infty$. Then $\mathfrak{m}=e^{-V} \operatorname{Vol}_{\mathrm{g}}$ for some locally bounded function $V$.

In the next theorem, we prove that there is no non-trivial measure other than volume measure on a $n$-dimensional Riemannian manifold, such that the corresponding metric measure space satisfies $\operatorname{CD}(k, n)$. On a weighted Riemannian manifold with $C^{2}$ weight, it is known that the Bakry-Émery condition $\operatorname{BE}(k, n)$ holds if and only if the weight is a constant. However in general case this is still an open problem. Recently De Philippis-Gigli [14] propose two definitions of non-collapsed metric measure space. We say that a $\operatorname{CD}(K, N)$ space $(X, \mathrm{~d}, \mathfrak{m})$ is weakly non-collapsed if $\mathfrak{m} \ll \mathcal{H}^{N}$, and we call it (strongly) non-collapsed if $\mathfrak{m}=c \mathcal{H}^{N}$ for some constant 
c. In case $X$ is a Riemannian manifold, the following Theorem tells us that noncollapsing and weakly non-collapsing are equivalent. We remark that this result is also obtained by Kapovitch-Ketterer in [22] and Kapovitch-Kell-Ketterer in [21], as a by-product in studying RCD condition on Alexandrov spaces.

Theorem 1.4 (Measure Rigidity: non-collapsed spaces, Theorem 3.6). Let (M,g) be a n-dimensional Riemannian manifold. Assume there exists a measure $\mathfrak{m}$ with full support such that $\left(M, \mathrm{~d}_{\mathrm{g}}, \mathfrak{m}\right)$ is $\mathrm{CD}(k, n)$. Then $\mathfrak{m}=c V_{o l}$ for some constant $c>0$.

In the last theorem, we study dimension-free $\mathrm{CD}(K, \infty)$ condition on manifolds with possibly non-smooth boundary. We prove that the reference measure must be supported on a geodesically convex set, and we answer the question why there is no mass on the boundary. In particular, we fully understand the curvaturedimension condition on smooth metric measure space with boundary (c.f. Theorem 2.4). Thus this result enhance our understanding to Cordero-Erausquin-McCannSchmuckenschläger's Riemannian interpolation inequality [13].

In attacking this problem, we assume neither infinitesimally Hilbertian nor nonbranching property, which are often used in the study of related problems. In particular, we do not know a priori whether the $L^{2}$-Wasserstein geodesic is unique or not (i.e. the Brenier-McCann's theorem). In the proof, we make full use of measure decomposition theory, $L^{1}$-optimal transport theory and its connection with $L^{2}$-optimal transport, which are developed by Klartag, Cavalletti and Mondino (c.f. [24], [9], [11]).

Theorem 1.5 (Measure rigidity: $\operatorname{CD}(K, \infty)$ condition, Theorem 3.7). Let $(M, \mathrm{~g})$ be a complete Riemannian manifold, $\Omega \subset M$ be an open set with Lipschitz boundary. Let $\mathrm{d}_{\Omega}$ be the intrinsic distance induced by Riemannian distance $\mathrm{d}_{\mathrm{g}}$ on $\bar{\Omega}$, and $\mathfrak{m}$ be a reference measure with supp $\mathfrak{m}=\bar{\Omega}$. Assume that $\partial \Omega$ is $C^{2}$ out of a $\mathcal{H}^{n-1}$-negligible set, and $\left(\bar{\Omega}, \mathrm{d}_{\Omega}, \mathfrak{m}\right)$ satisfies $\operatorname{CD}(K, \infty)$ condition for some $K \in \mathbb{R}$, then we have the following rigidity results.

1) $\bar{\Omega}$ is g-geodesically convex, that is, any shortest path in $\left(\bar{\Omega}, \mathrm{d}_{\Omega}\right)$ is a geodesic segment in $(M, \mathrm{~g})$;

2) $\left.\mathfrak{m}\right|_{\partial \Omega}=0$ and $\mathfrak{m}=e^{-V} \operatorname{Vol}_{\mathrm{g}}$ for some semi-convex, locally Lipschitz function $V$

3) $\left(\bar{\Omega}, \mathrm{d}_{\Omega}, \mathfrak{m}\right)$ is a $\operatorname{RCD}(K, \infty)$ space.

In particular, $\left(\bar{\Omega}, \mathrm{d}_{\Omega}, \operatorname{Vol}_{\mathrm{g}}\right)$ is $\operatorname{CD}(K, \infty)$ if and only if $\bar{\Omega}$ is g-geodesically convex and Ricci $\geq K$ on $\Omega$.

At last, we remark that most of the measure rigidity results obtained in this paper are still true on Alexandrov spaces with bounded curvature, which can be proved in similar ways. Compared with previous results about curvature-dimension conditions with finite dimension, we use some new methods to deal with the infinite dimensional problem. We believe that these methods have potential applications on more general metric measure spaces. 
Acknowledgement: This research is part of a project which has received funding from the European Research Council (ERC) under the European Union's Horizon 2020 research and innovation programme (grant agreement No. 637851). Part of Section 2 had been finished when the author was supported by a postdoctoral fellowship from Hausdorff Center for Mathematics (HCM) in Bonn. The author wants to thank E. Milman and M. Kell for helpful discussions and comments, also the anonymous referee for the valuable report which improved readability.

\section{Smooth metric measure spaces with boundary}

Let $(X, \mathrm{~d}, \mathfrak{m})$ be an abstract metric measure space. We say that $f \in L^{2}(X, \mathfrak{m})$ is a Sobolev function in $W^{1,2}(X, \mathrm{~d}, \mathfrak{m})$ if there exists a sequence of Lipschitz functions $\left(f_{n}\right)_{n} \subset L^{2}$, such that $f_{n} \rightarrow f$ and $\operatorname{lip}\left(f_{n}\right) \rightarrow G$ in $L^{2}$ for some $G$, where $\operatorname{lip}\left(f_{n}\right)$ is the local Lipschitz constant of $f_{n}$ defined by

$$
\operatorname{lip}\left(f_{n}\right)(x):=\varlimsup_{y \rightarrow x} \frac{\left|f_{n}(y)-f_{n}(x)\right|}{\mathrm{d}(y, x)}
$$

(and 0 if $x$ is an isolated point). It is known that there exists a minimal function in $\mathfrak{m}$-a.e. sense, denoted it by $|\mathrm{D} f|$, called minimal weak upper gradient. If $(X, \mathrm{~d})$ is a Riemannian manifold and $\mathfrak{m}$ is the volume measure, we know that $|\mathrm{D} f|=|\nabla f|=$ $\operatorname{lip}(f)$ for any $f \in C^{\infty}$ (c.f. Theorem $6.1[12]$ ). Furthermore, let $\Omega \subset X$ be an open set with $\mathfrak{m}(\partial \Omega)=0$, by locality we have $|\mathrm{D} f|_{\bar{\Omega}}=|\nabla f| \mathfrak{m}$-a.e..

We equip $W^{1,2}(X, \mathrm{~d}, \mathfrak{m})$ with the norm

$$
\|f\|_{W^{1,2}(X, \mathrm{~d}, \mathfrak{m})}:=\sqrt{\|f\|_{L^{2}(X, \mathfrak{m})}^{2}+\||\mathrm{D} f|\|_{L^{2}(X, \mathfrak{m})}^{2}} .
$$

It is known that $W^{1,2}(X, \mathrm{~d}, \mathfrak{m})$ is a Banach space, but not necessarily a Hilbert space. We say that $(X, \mathrm{~d}, \mathfrak{m})$ is an infinitesimally Hilbertian space if $W^{1,2}$ is a Hilbert space. Obviously, Riemannian manifolds equipped with volume measure are infinitesimally Hilbertian spaces. In general, infinitesimal Hilbertianity is not trivial even if the base space $X$ is a manifold.

On an infinitesimally Hilbertian space, there a pointwise bilinear map defined by

$$
\left[W^{1,2}\right]^{2} \ni(f, g) \mapsto\langle\nabla f, \nabla g\rangle:=\frac{1}{4}\left(|\mathrm{D}(f+g)|^{2}-|\mathrm{D}(f-g)|^{2}\right) .
$$

It can be seen that $\langle\cdot, \cdot\rangle=\mathrm{g}(\cdot, \cdot)$ on a Riemannian manifold $(M, \mathrm{~g})$.

Then we can define the measure-valued Laplacian via integration by part.

Definition 2.1 (Measure-valued Laplacian, $[16,17])$. The space $\mathrm{D}(\boldsymbol{\Delta}) \subset W^{1,2}(X, \mathrm{~d}, \mathfrak{m})$ is the space of $f \in W^{1,2}(X, \mathrm{~d}, \mathfrak{m})$ such that there is a measure $\mu$ satisfying

$$
\int h \mathrm{~d} \mu=-\int\langle\nabla h, \nabla f\rangle \mathrm{d} \mathfrak{m}, \quad \forall h \text { Lipschitz with bounded support. }
$$

In this case the measure $\mu$ is unique and we shall denote it by $\Delta f$. If $\Delta f \ll m$, we denote its density by $\Delta f$. 
The following proposition links the curvature-dimension condition $\operatorname{RCD}(K, \infty)$ and the non-smooth Bakry-Émery theory. We say that a space is $\operatorname{RCD}(K, \infty)$ if it is a $\mathrm{CD}(K, \infty)$ space defined by Lott-Sturm-Villani in [25,30,31], equipped with an infinitesimally Hilbertian Sobolev space. For more details, see [7] (also [5]).

We define $\operatorname{TestF}(X, \mathrm{~d}, \mathfrak{m}) \subset W^{1,2}(X, \mathrm{~d}, \mathfrak{m})$, the set of test functions by

$\operatorname{TestF}(X, \mathrm{~d}, \mathfrak{m}):=\left\{f \in \mathrm{D}(\boldsymbol{\Delta}) \cap L^{\infty}:|\mathrm{D} f| \in L^{\infty}\right.$ and $\left.\Delta f \in W^{1,2}(X, \mathrm{~d}, \mathfrak{m}) \cap L^{\infty}(X, \mathfrak{m})\right\}$.

It is known (c.f. [29]) that $\operatorname{TestF}(X, \mathrm{~d}, \mathfrak{m})$ is dense in $W^{1,2}(X, \mathrm{~d}, \mathfrak{m})$ when $(X, \mathrm{~d}, \mathfrak{m})$ is $\operatorname{RCD}(K, \infty)$.

Let $f, g \in \operatorname{TestF}(X, \mathrm{~d}, \mathfrak{m})$. It is known from [29] that measure $\boldsymbol{\Gamma}_{2}(f, g)$ is welldefined by

$$
\Gamma_{2}(f, g)=\frac{1}{2} \Delta\langle\nabla f, \nabla g\rangle-\frac{1}{2}(\langle\nabla f, \nabla \Delta g\rangle+\langle\nabla g, \nabla \Delta f\rangle) \mathfrak{m},
$$

and we put $\boldsymbol{\Gamma}_{2}(f):=\boldsymbol{\Gamma}_{2}(f, f)$. Then we have the following Bochner inequality on metric measure spaces.

Proposition 2.2 (Bakry-Émery condition, $[7,8])$. Let $(X, \mathrm{~d}, \mathfrak{m})$ be a $\operatorname{RCD}(K, \infty)$ space for some $K \in \mathbb{R}$. Then

$$
\Gamma_{2}(f) \geq K|\mathrm{D} f|^{2} \mathfrak{m}
$$

for any $f \in \operatorname{TestF}(X, \mathrm{~d}, \mathfrak{m})$.

Let $f \in \operatorname{TestF}(X, \mathrm{~d}, \mathfrak{m})$. The Hessian map $\operatorname{Hess}_{f}:\{\nabla g: g \in \operatorname{TestF}(X, \mathrm{~d}, \mathfrak{m})\}^{2} \mapsto$ $L^{0}(X, \mathrm{~d}, \mathfrak{m})$ is defined by

$$
2 \operatorname{Hess}_{f}(\nabla g, \nabla h)=\langle\nabla g, \nabla\langle\nabla f, \nabla h\rangle\rangle+\langle\nabla h, \nabla\langle\nabla f, \nabla g\rangle\rangle-\langle\nabla f, \nabla\langle\nabla g, \nabla h\rangle\rangle
$$

for any $g, h \in \operatorname{TestF}(X, \mathrm{~d}, \mathfrak{m})$. t can be proved (see Theorem 3.4 [29] and Theorem 3.3.8 [17]) that $\operatorname{Hess}_{f}(\cdot, \cdot)$ can be extended to a symmetric $L^{\infty}(X, \mathrm{~d}, \mathfrak{m})$-bilinear map on the $L^{2}$-tangent module of $(X, \mathrm{~d}, \mathfrak{m})$, and is continuous with values in $L^{0}(X, \mathrm{~d}, \mathfrak{m})$. On Riemannian manifolds, Hess $f$ coincides with $\mathrm{D}^{2} f$, which is the Hessian (tensor) in classical sense.

Furthermore, we have the following proposition, due to Gigli [17].

Proposition 2.3 (Theorem 3.6.7 [17]). Let $M$ be a $\operatorname{RCD}(K, \infty)$ space. Then

$$
\operatorname{Ricci}(\nabla f, \nabla f) \geq K|\mathrm{D} f|^{2} \mathfrak{m}
$$

for any $f \in \operatorname{TestF}(X, \mathrm{~d}, \mathfrak{m})$, where the measure-valued Ricci tensor Ricci is defined by

$$
\boldsymbol{\operatorname { R i c c i }}(\nabla f, \nabla f):=\boldsymbol{\Gamma}_{2}(f)-\left\|\operatorname{Hess}_{f}\right\|_{\mathrm{HS}}^{2} \mathfrak{m} .
$$

Now we introduce our first theorem. 
Theorem 2.4 (Measure-valued Ricci tensor). Let $\left(M, \mathrm{~g}, e^{-V} \mathrm{Vol}_{\mathrm{g}}\right)$ be a n-dimensional weighted Riemannian manifold and $\Omega \subset M$ be a connected open set with $(n-1)$ dimensional smooth orientable boundary. Then the measure valued Ricci tensor on $\left(\bar{\Omega}, \mathrm{d}_{\Omega}, e^{-V} \operatorname{Vol}_{\mathrm{g}}\right)$ is given by

$$
\operatorname{Ricci}_{\Omega}(\nabla g, \nabla g)=\left.\operatorname{Ricci}_{V}(\nabla g, \nabla g) e^{-V} \mathrm{dVol}_{g}\right|_{\Omega}+\left.I I(\nabla g, \nabla g) e^{-V} \mathrm{~d} \mathcal{H}^{n-1}\right|_{\partial \Omega}
$$

for any $g \in C_{c}^{\infty}$ with $\mathrm{g}(\mathrm{N}, \nabla g)=0$, where $\mathrm{N}$ is the outward normal vector field on $\partial \Omega$, and Ricci $_{V}$ is the Bakry-Emery's generalized Ricci tensor.

Proof. From integration by part formula (Green's formula) on a Riemannian manifold, we know

$$
\int \mathrm{g}(\nabla f, \nabla g) e^{-V} \mathrm{dVol}_{\mathrm{g}}=-\int f \Delta_{V} g e^{-V} \mathrm{dVol}_{\mathrm{g}}+\left.\int_{\partial \Omega} f \mathrm{~g}(\mathrm{~N}, \nabla g) e^{-V} \mathrm{~d} \mathcal{H}^{n-1}\right|_{\partial \Omega}
$$

for any $f, g \in C_{c}^{\infty}$, where $\Delta_{V}:=(\Delta-\nabla V)$ and $\left.\mathcal{H}^{n-1}\right|_{\partial \Omega}$ is the $(n-1)$-dimensional Hausdorff measure on $\partial \Omega$.

Hence $g \in \mathrm{D}\left(\boldsymbol{\Delta}_{\Omega}\right)$ and the measure-valued Laplacian is given by the following formula

$$
\Delta_{\Omega} g=\left.\Delta_{V} g e^{-V} \operatorname{Vol}_{g}\right|_{\Omega}-\left.\mathrm{g}(\mathrm{N}, \nabla g) e^{-V} \mathcal{H}^{n-1}\right|_{\partial \Omega} .
$$

Therefore for any $g \in C_{c}^{\infty}$ with $g(\mathrm{~N}, \nabla g)=0$ on $\partial \Omega$, we have $g \in \operatorname{TestF}(\Omega)$.

Now we can compute the measure-valued Ricci tensor. Let $g \in C_{c}^{\infty}$ be a test function with $g(\mathrm{~N}, \nabla g)=0$ on $\partial \Omega$. We have

$$
\begin{aligned}
& \operatorname{Ricci}_{\Omega}(\nabla g, \nabla g)= \frac{1}{2} \Delta_{\Omega}|\mathrm{D} g|_{\Omega}^{2}-\left\langle\nabla g, \nabla \Delta_{\Omega} g\right\rangle_{\Omega} e^{-V} \operatorname{Vol}_{\mathrm{g}}-\left\|\operatorname{Hess}_{g}\right\|_{\mathrm{HS}}^{2} e^{-V} \operatorname{Vol}_{\mathrm{g}} \\
&= \frac{1}{2} \Delta_{V}|\nabla g|^{2} e^{-V} \operatorname{Vol}_{\mathrm{g}}-\mathrm{g}\left(\nabla g, \nabla \Delta_{V} g\right) e^{-V} \operatorname{Vol}_{\mathrm{g}}-\left\|\operatorname{Hess}_{g}\right\|_{\mathrm{HS}}^{2} e^{-V} \operatorname{Vol}_{\mathrm{g}} \\
&-\left.\frac{1}{2} \mathrm{~g}\left(\mathrm{~N}, \nabla|\nabla g|^{2}\right) e^{-V} \mathcal{H}^{n-1}\right|_{\partial \Omega} \\
&= \operatorname{Ricci}(\nabla g, \nabla g) e^{-V} \operatorname{Vol}_{\mathrm{g}}+\operatorname{Hess}_{V}(\nabla g, \nabla g) e^{-V} \operatorname{Vol}_{\mathrm{g}} \\
& \quad-\left.\frac{1}{2} \mathrm{~g}\left(\mathrm{~N}, \nabla|\nabla g|^{2}\right) e^{-V} \mathcal{H}^{n-1}\right|_{\partial \Omega} \\
&= \operatorname{Ricci}_{V}(\nabla g, \nabla g) e^{-V} \operatorname{Vol}_{\mathrm{g}}-\left.\frac{1}{2} \mathrm{~g}\left(\mathrm{~N}, \nabla|\nabla g|^{2}\right) e^{-V} \mathcal{H}^{n-1}\right|_{\partial \Omega},
\end{aligned}
$$

where we use Bochner's formula at the third equality, and $\operatorname{Ricci}_{V}=$ Ricci $+\operatorname{Hess}_{V}$ is Bakry-Émery's generalized Ricci tensor on weighted Riemannian manifold with weight $e^{-V}$.

By definition of second fundamental form, we have

$$
I I(\nabla g, \nabla g)=\mathrm{g}\left(\nabla_{\nabla g} \mathrm{~N}, \nabla g\right)=\mathrm{g}(\nabla \mathrm{g}(\mathrm{N}, \nabla g), \nabla g)-\frac{1}{2} \mathrm{~g}\left(\mathrm{~N}, \nabla|\nabla g|^{2}\right) .
$$

Recall that $\mathrm{g}(N, \nabla g)=0$ on $\partial \Omega$, we have $\mathrm{g}\left(\nabla_{\nabla g} \mathrm{~N}, \nabla g\right)=-\frac{1}{2} \mathrm{~g}\left(\mathrm{~N}, \nabla|\nabla g|^{2}\right)$.

Finally, we obtain

$$
\operatorname{Ricci}_{\Omega}(\nabla g, \nabla g)=\operatorname{Ricci}_{V}(\nabla g, \nabla g) \operatorname{Vol}_{g}+\left.I I(\nabla g, \nabla g) e^{-V} \mathcal{H}_{n-1}\right|_{\partial \Omega}
$$

for any $g \in C_{c}^{\infty}$ with $g(N, \nabla g)=0$. 
At the last, we list some simple applications of Theorem 2.4 whose proof can be found in [19], see also Theorem 1.2.1 and Theorem 3.3.2 in [34] for more details.

Corollary 2.5 (Rigidity: convexity of the boundary ). Let $\left(\bar{\Omega}, \mathrm{d}_{\Omega}, e^{-V} \operatorname{Vol}_{\mathrm{g}}\right)$ be a space as stated in Theorem 2.4. Then it is $\operatorname{RCD}(K, \infty)$ if and only if $\partial \Omega$ is convex and $\operatorname{Ricci}_{V} \geq K$ on $\Omega$.

The next result tells us that the boundary does not influence the dimension bound of the smooth metric measure space.

Corollary 2.6. A n-dimensional Riemannian manifold with boundary is $\operatorname{RCD}(K, \infty)$ if and only if it is $\operatorname{RCD}(K, n)$.

The last corollary characterizes metric measure spaces with upper Ricci curvature bound, see also [15] for a rigidity result concerning a different notion of upper Ricci bound.

Corollary 2.7 (c.f. [26] p.104). If $\mathbf{R i c c i}_{\Omega} \ll \operatorname{Vol}_{\mathrm{g}}$, then $\partial \Omega$ is totally geodesic, i.e. $I I \equiv 0$.

\section{Main results: measure rigidity theorems}

At first, we recall some fundamental properties of spaces satisfying Lott-SturmVillani's synthetic lower Ricci curvature bound (including $\operatorname{CD}(K, \infty), \operatorname{CD}(K, N)$ and $\operatorname{RCD}(K, \infty)$ spaces $)$.

1) (Uniform density bound of intermediate measures on $\operatorname{CD}(K, \infty)$ spaces, Lemma 3.1 [27].) Let $\mu_{0}, \mu_{1} \in \mathcal{P}(X)$ be a pair of probability measures with bounded density and so that $W_{2}\left(\mu_{0}, \mu_{1}\right)<\infty$. Suppose also that $\operatorname{diam}\left(\operatorname{supp} \mu_{0} \cup\right.$ $\left.\operatorname{supp} \mu_{1}\right)<\infty$. Then there exists a $L^{2}$-Wasserstein geodesic $\left(\mu_{t}\right)$ connecting $\mu_{0}$ and $\mu_{1}$ such that the densities $\left\{\frac{\mathrm{d} \mu_{t}}{\mathrm{~d} \mathfrak{m}}\right\}_{t}$ are uniformly bounded.

2) (Generalized Brunn-Minkowski inequality on $\mathrm{CD}(K, N)$ spaces, Proposition 2.1 [31].) Given $K, N \in \mathbb{R}$, with $N \geq 1$, we set for $(t, \theta) \in[0,1] \times \mathbb{R}^{+}$,

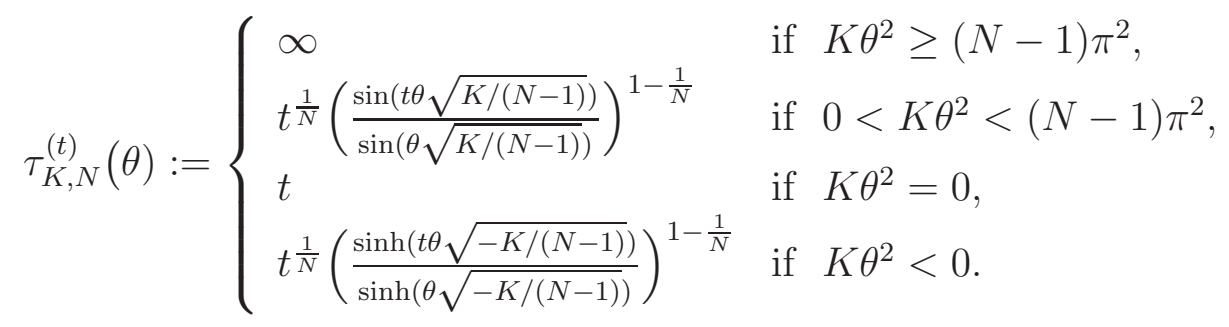

Then for any measurable sets $A_{0}, A_{1} \subset X$ with $\mathfrak{m}\left(A_{0}\right)+\mathfrak{m}\left(A_{1}\right)>0, t \in[0,1]$ and $N^{\prime} \geq N$, we have

$$
\mathfrak{m}\left(A_{t}\right)^{\frac{1}{N^{\prime}}} \geq \tau_{K, N^{\prime}}^{(1-t)}(\Theta) \mathfrak{m}\left(A_{0}\right)^{\frac{1}{N^{\prime}}}+\tau_{K, N^{\prime}}^{(t)}(\Theta) \mathfrak{m}\left(A_{1}\right)^{\frac{1}{N^{\prime}}},
$$

where $A_{t}$ denotes the set of points which divide geodesics starting in $A_{0}$ and ending in $A_{1}$ with ratio $\frac{t}{1-t}$ and where $\Theta$ denotes the minimal $(K \geq 0)$ or maximal $(K<0)$ length of such geodesics. In particular, when $A_{0}$ is a single point, 
we have the following $(K, N)$-measure contraction property (or $\operatorname{MCP}(K, N)$ condition for short):

$$
\mathfrak{m}\left(A_{t}\right) \geq\left[\tau_{K, N}^{(t)}(\Theta)\right]^{N} \mathfrak{m}\left(A_{1}\right)
$$

3) (Riemannian-Curvature-Dimension condtion, i.e. RCD condition, see [7]) We say that a space is $\operatorname{RCD}(K, \infty)$ (or $\operatorname{RCD}(K, N)$ ) if it is an infinitesimally Hilbertian $\mathrm{CD}(K, \infty)$ (or $\mathrm{CD}(K, N)$ respectively) space. It is known that Riemannian manifolds with lower Ricci curvature bound, Ricci limit spaces, and Alexandrov spaces with lower curvature bound are RCD spaces.

[20, Proposition 3.1, Part a)]

Let $(X, \mathrm{~d}, \mathfrak{m})$ be a $\operatorname{RCD}(K, \infty)$ space. For any $L^{2}$-Wasserstein geodesic $\left(\mu_{t}\right)$ connecting $\mu_{0}, \mu_{1} \ll \mathfrak{m}$, there exists $\Pi \in \mathcal{P}(\operatorname{Geo}(\mathrm{X}, \mathrm{d}))$ such that $\left(e_{t}\right)_{\sharp} \Pi=\mu_{t}$ (c.f. Theorem 2.10 [4]). By essentially non-branching property of $\operatorname{RCD}(K, \infty)$ spaces (c.f. [28]), $\Pi$ is concentrated on a set of non-branching geodesics, where we say that a set $\Gamma \subset \operatorname{Geo}(\mathrm{X}, \mathrm{d})$ is non-branching if for any $\gamma^{1}, \gamma^{2} \in \Gamma$, it holds:

$$
\exists t \in(0,1) \text { s.t. } \forall s \in[0, t] \gamma_{t}^{1}=\gamma_{t}^{2} \Rightarrow \gamma_{s}^{1}=\gamma_{s}^{2}, \forall s \in[0,1] .
$$

Furthermore, there exits a unique $L^{2}$-Wasserstein geodesic connecting $\mu_{0}, \mu_{1}$ (c.f. [28]) which is induced by an optimal transport map.

\subsection{Measure rigidity: absolute continuity}

Let $\left(X, \mathrm{~d}, \mathfrak{m}_{1}\right)$ and $\left(X, \mathrm{~d}, \mathfrak{m}_{2}\right)$ be two metric measure spaces satisfying essentially non-branching $\operatorname{MCP}(K, N)$ condition. For $N<\infty$, Cavalletti and Mondino (c.f. Proposition 8.1 and Corollary 8.2 in [10]) prove the mutual absolute continuity of the reference measures $\mathfrak{m}_{1}, \mathfrak{m}_{2}$ (see [23] for a different proof given by Kell). In case $X$ is a Riemannian manifold, we extend such result to dimension-free $\operatorname{CD}(K, \infty)$ condition and prove a quantitative density estimate under $\operatorname{CD}(K, N)$ condition.

Proposition 3.1 (Measure rigidity: absolute continuity). Let ( $M, \mathrm{~g}, \mathrm{Vol}_{\mathrm{g}}$ ) be a complete $n$-dimensional Riemannian manifold, $\mathrm{d}_{\mathrm{g}}$ be the Riemannian distance induced by $\mathrm{g}$ and $\mathfrak{m}$ be a Borel measure with full support on $M$. Then we have the following results.

a) Assume that $\left(M, \mathrm{~d}_{\mathrm{g}}, \mathfrak{m}\right)$ is $\mathrm{CD}(K, \infty)$ for some $K \in \mathbb{R}$. Then $\mathfrak{m} \ll \operatorname{Vol}_{\mathrm{g}}$.

b) Assume that $\left(M, \mathrm{~d}_{\mathrm{g}}, \mathfrak{m}\right)$ is $\operatorname{MCP}(K, N)$ for some $K \in \mathbb{R}, N<\infty$. Then we have

$$
\frac{\mathfrak{m}\left(B_{r}(x)\right)}{\operatorname{Vol}_{\mathrm{g}}\left(B_{r}(x)\right)} \in L_{\mathrm{loc}}^{\infty} \quad \text { uniformly in } r>0 \text {. }
$$

In particular, $\mathfrak{m}=e^{-V} \operatorname{Vol}_{\mathrm{g}}$ for some $e^{-V} \in L_{\mathrm{loc}}^{\infty}$.

c) Assume that $\left(M, \mathrm{~d}_{g}, \mathfrak{m}\right)$ is $\operatorname{MCP}(K, N)$ for some $K \in \mathbb{R}, N<\infty$. Then we have

$$
\frac{r^{N-n} \operatorname{Vol}_{\mathrm{g}}\left(B_{r}(x)\right)}{\mathfrak{m}\left(B_{r}(x)\right)} \in L_{\mathrm{loc}}^{\infty} \quad \text { uniformly in } r>0 \text {. }
$$

In particular, when $N=n$, we know $\mathfrak{m}=e^{-V} \operatorname{Vol}_{\mathrm{g}}$ for some $V \in L_{\mathrm{loc}}^{\infty}$. 
Proof. Part a):

Let $U \subset M$ be a bounded and geodesically convex open set. By local finiteness of $\mathfrak{m}$ (c.f. Theorem 4.24 [30]) we know $\mathfrak{m}(U)<\infty$. By definition, $\left(\bar{U}, \mathrm{~d}_{\mathrm{g}},\left.\mathfrak{m}\right|_{U}\right)$ is still a $\mathrm{CD}(K, \infty)$ space.

Let $\mathfrak{m}:=\mathfrak{m}_{\mathrm{ac}}+\mathfrak{m}_{\mathrm{s}}$ be the Lebesgue decomposition of $\mathfrak{m}$ with respect to the Riemannian volume measure $\operatorname{Vol}_{\mathrm{g}}$. We will prove $\mathfrak{m} \ll \operatorname{Vol}_{\mathrm{g}}$ in the following two steps. Firstly, we will show $\mathfrak{m}_{\mathrm{ac}} \neq 0$. Then we will prove $\mathfrak{m}_{\mathrm{s}}=0$.

Step 1: $\mathfrak{m}_{\mathrm{ac}} \neq 0$ on $U$.

Given a parameter $r>0$, we define $\epsilon_{r}: U \mapsto \mathbb{R}^{+}$by

$$
\epsilon_{r}(x):=\frac{\mathfrak{m}\left(B_{r}(x)\right)}{\operatorname{Vol}_{\mathrm{g}}\left(B_{r}(x)\right)} .
$$

Assume by contradiction that $\mathfrak{m}=\mathfrak{m}_{\mathrm{s}}$, then for any constant $c>0$, there must be

$$
\mathfrak{m}\left(\left\{x \in U: \underline{\lim _{r \rightarrow 0}} \epsilon_{r}(x) \leq c\right\}\right)=0,
$$

otherwise we can prove $\mathfrak{m}_{\mathrm{ac}} \neq 0$ by a standard measure-theoretic argument (using covering theorems). For any $(c, r) \in(0,+\infty) \times(0,1)$, we define the set $S(c, r) \subset U$ by

$$
S(c, r):=\left\{x \in U: \epsilon_{s}(x) \geq c \text { for all } s \leq r\right\} .
$$

From definition, we have the following monotonicity

$$
S\left(c, r_{2}\right) \subset S\left(c, r_{1}\right) \text { for all } r_{1}<r_{2} \text {. }
$$

It can also be seen that

$$
\left\{x \in U: \varliminf_{r \rightarrow 0} \epsilon_{r}(x)>c\right\} \subset \cup_{0<r<1} S(c, r)=\lim _{r \rightarrow 0} S(c, r) .
$$

So by (3.4), we obtain

$$
\mathfrak{m}\left(\lim _{r \rightarrow 0} S(c, r)\right)=\lim _{r \rightarrow 0} \mathfrak{m}(S(c, r))=\mathfrak{m}(U) .
$$

Combining (3.5), (3.6), and the assumption supp $\mathfrak{m}=X$, we get

$$
\lim _{r \rightarrow 0} \operatorname{Vol}_{\mathrm{g}}(\overline{S(c, r)})=\operatorname{Vol}_{\mathrm{g}}(U) .
$$

Next we will deduce contradiction from (3.7) and $\operatorname{CD}(K, \infty)$ condition.

Fix a point $y_{0} \in U$ with $\lim _{r \rightarrow 0} \epsilon_{r}\left(y_{0}\right)=\infty$. By Rauch's (and Toponogov's) comparison theorem, there exists a small $R>0$ and constants $c_{1}, c_{2}>0$ such that

$$
c_{1} t \mathrm{~d}_{\mathrm{g}}(y, z)<\mathrm{d}_{\mathrm{g}}\left(\gamma_{t}^{x z}, \gamma_{t}^{x y}\right)<c_{2} t \mathrm{~d}_{\mathrm{g}}(y, z) \quad \forall t \in(0,1], \quad \forall x, y, z \in B_{3 R}\left(y_{0}\right),
$$

and

$$
\mathrm{d}_{\mathrm{g}}\left(\gamma_{s}^{x z}, \gamma_{t}^{x y}\right)>c_{1} s \wedge t \mathrm{~d}_{\mathrm{g}}(y, z) \quad \forall s, t \in(0,1)
$$


for any $x, y, z \in B_{3 R}\left(y_{0}\right)$ with $\mathrm{d}_{\mathrm{g}}(x, y)=\mathrm{d}_{\mathrm{g}}(x, z)$, where $\gamma^{x z}$ denotes the geodesic from $x$ to $z$ and $\gamma^{x y}$ denotes the geodesic from $x$ to $y$. Moreover, the following comparison principle holds for any geodesic $\gamma^{1}, \gamma^{2}$ with endpoints in $B_{3 R}\left(y_{0}\right) \subset U$ :

$$
\mathrm{d}_{\mathrm{g}}\left(\gamma_{t}^{1}, \gamma_{t}^{2}\right)<c_{2} \max \left\{\mathrm{d}_{\mathrm{g}}\left(\gamma_{0}^{1}, \gamma_{0}^{2}\right), \mathrm{d}_{\mathrm{g}}\left(\gamma_{1}^{1}, \gamma_{1}^{2}\right)\right\} \quad \forall t \in(0,1)
$$

Let $y \in B_{3 R}\left(y_{0}\right) \backslash \overline{B_{2 R}\left(y_{0}\right)}$. Consider the $L^{2}$-Wasserstein geodesic $\left(\mu_{t}^{r, y}\right)_{t}$ from $\mu_{0}^{r, y}:=\left.\frac{1}{\mathfrak{m}\left(B_{r}\left(y_{0}\right)\right)} \mathfrak{m}\right|_{B_{r}\left(y_{0}\right)}$ to $\mu_{1}^{r, y}:=\left.\frac{1}{\mathfrak{m}\left(B_{r}(y)\right)} \mathfrak{m}\right|_{B_{r}(y)}$. By density bound of intermediate measures on $\mathrm{CD}(K, \infty)$ space (c.f. Lemma $3.1[27]$ ), we get the following (uniform) estimate

$$
\mathfrak{m}\left(\operatorname{supp} \mu_{t}^{r, y}\right) \gtrsim \min \left\{\mathfrak{m}\left(B_{r}\left(y_{0}\right)\right), \mathfrak{m}\left(B_{r}(y)\right)\right\} \quad \forall t \in[0,1],
$$

where we adopt the notation $A \lesssim B$ if there is a constant $C>0$ such that $A<C B$. Combining (3.11) and the fact $\operatorname{Vol}_{\mathrm{g}}\left(B_{r}\right) \gtrsim r^{n}$, we get

$$
\mathfrak{m}\left(\operatorname{supp} \mu_{t}^{r, y}\right) \gtrsim r^{n} \min \left\{\epsilon_{r}\left(y_{0}\right), \epsilon_{r}(y)\right\} \quad \forall t \in[0,1] .
$$

Let $T_{t}$ be the optimal transport map which induces $\left(\mu_{t}^{r, y}\right)_{t}$. By (3.10) we know

$$
\mathrm{d}_{\mathrm{g}}\left(T_{t}(x), \gamma_{t}^{y_{0} y}\right) \leq c_{2} \max \left\{\mathrm{d}_{\mathrm{g}}\left(x, y_{0}\right), \mathrm{d}_{\mathrm{g}}\left(T_{1}(x), y\right)\right\} \leq c_{2} r
$$

for any $x \in B_{r}\left(y_{0}\right)$, where $\gamma^{y_{0} y}$ is the geodesic from $y_{0}$ to $y$. Therefore

$$
\mu_{t}^{r, y}\left(B_{c_{2} r}\left(\gamma_{t}^{y_{0} y}\right)\right)=1 \quad \forall t \in[0,1] .
$$

In particular, $\cup_{t} \operatorname{supp} \mu_{t}^{r, y} \subset \overline{\left(\gamma^{y_{0} y}\right)_{c_{2} r}}$, where $\left(\gamma_{t}^{y_{0} y}\right)_{c_{2} r}$ is the $c_{2} r$-neighbourhood of $\gamma^{y_{0} y}$ w.r.t. Hausdorff distance.

For any $c>0$, by (3.7), there exists a small $r=r(c) \ll R$ such that

$$
\frac{\operatorname{Vol}_{\mathrm{g}}\left(\overline{\left\{\epsilon_{r} \geq c\right\}} \cap B_{3 R}\left(y_{0}\right) \backslash B_{2 R}\left(y_{0}\right)\right)}{\operatorname{Vol}_{\mathrm{g}}\left(B_{3 R}\left(y_{0}\right) \backslash B_{2 R}\left(y_{0}\right)\right)}>\frac{1}{2} .
$$

Consider the projection map Prj: $B_{3 R}\left(y_{0}\right) \mapsto \partial B_{2 R}\left(y_{0}\right)$ along the radius, defined by

$$
\operatorname{Prj}(x):=\exp _{y_{0}}\left(\frac{2 R \exp _{y_{0}}^{-1}(x)}{\left|\exp _{y_{0}}^{-1}(x)\right|}\right)
$$

It is known that

$$
\operatorname{Vol}_{\mathrm{g}}\left(B_{3 R}\left(y_{0}\right) \backslash B_{2 R}\left(y_{0}\right)\right)=\int_{2 R}^{3 R} \mathcal{H}^{n-1}\left(\partial B_{t}\left(y_{0}\right)\right) \mathrm{d} t .
$$

Hence by Fubini's theorem and (3.14), there is $t_{0} \in[2 R, 3 R]$ such that

$$
\mathcal{H}^{n-1}\left(\overline{\left\{\epsilon_{r} \geq c\right\}} \cap \partial B_{t_{0}}\left(y_{0}\right)\right)>\frac{1}{2} \mathcal{H}^{n-1}\left(\partial B_{t_{0}}\left(y_{0}\right)\right) .
$$


In addition, by (3.8), we have

$$
\left.\mathcal{H}^{n-1}\right|_{\partial B_{2 R}\left(y_{0}\right)} \geq c_{1} \frac{2 R}{t_{0}}(\operatorname{Prj})_{\sharp}\left(\left.\mathcal{H}^{n-1}\right|_{\partial B_{t_{0}}\left(y_{0}\right)}\right) \geq \frac{2 c_{1}}{3}(\operatorname{Prj})_{\sharp}\left(\left.\mathcal{H}^{n-1}\right|_{\partial B_{t_{0}}\left(y_{0}\right)}\right)
$$

and

$$
\left.\mathcal{H}^{n-1}\right|_{\partial B_{2 R}\left(y_{0}\right)} \leq c_{2} \frac{t_{0}}{2 R}(\operatorname{Prj})_{\sharp}\left(\left.\mathcal{H}^{n-1}\right|_{\partial B_{t_{0}}\left(y_{0}\right)}\right) \leq \frac{3 c_{2}}{2}(\operatorname{Prj})_{\sharp}\left(\left.\mathcal{H}^{n-1}\right|_{\partial B_{t_{0}}\left(y_{0}\right)}\right)
$$

Combining the estimates (3.16), (3.17) and (3.18), we get

$$
\begin{aligned}
& \left.\mathcal{H}^{n-1}\right|_{\partial B_{2 R}\left(y_{0}\right)}\left(\operatorname{Prj}\left(\overline{\left\{\epsilon_{r} \geq c\right\}} \cap B_{3 R}\left(y_{0}\right) \backslash B_{2 R}\left(y_{0}\right)\right)\right) \\
\geq & \left.\mathcal{H}^{n-1}\right|_{\partial B_{2 R}\left(y_{0}\right)}\left(\operatorname{Prj}\left(\overline{\left\{\epsilon_{r} \geq c\right\}} \cap \partial B_{t_{0}}\left(y_{0}\right)\right)\right) \\
\text { by }(3.17) \geq & \left.\frac{2 c_{1}}{3} \mathcal{H}^{n-1}\right|_{\partial B_{t_{0}}\left(y_{0}\right)}\left(\overline{\left\{\epsilon_{r} \geq c\right\}} \cap \partial B_{t_{0}}\left(y_{0}\right)\right) \\
\text { by }(3.16)> & \frac{2 c_{1}}{3} \frac{1}{2} \mathcal{H}^{n-1}\left(\partial B_{t_{0}}\left(y_{0}\right)\right) \\
\text { by (3.18) } \geq & \frac{c_{1}}{3}\left(\operatorname{Prj}^{-1}\right)_{\sharp}\left(\left.\frac{2}{3 c_{2}} \mathcal{H}^{n-1}\right|_{\partial B_{2 R}\left(y_{0}\right)}\right)\left(\partial B_{t_{0}}\left(y_{0}\right)\right),
\end{aligned}
$$

and finally

$$
\mathcal{H}^{n-1}\left(\operatorname{Prj}\left(\overline{\left\{\epsilon_{r} \geq c\right\}} \cap B_{3 R}\left(y_{0}\right) \backslash B_{2 R}\left(y_{0}\right)\right)\right) \geq \frac{2}{9} \frac{c_{1}}{c_{2}} \mathcal{H}^{n-1}\left(\partial B_{2 R}\left(y_{0}\right)\right)
$$

By (3.19) we can find an integer $N \gtrsim \frac{1}{r^{n-1}}$ which is independent of $c$, and $N$ points $\left\{y_{1}, y_{2}, \ldots, y_{N}\right\} \subset\left\{x: \epsilon_{r}(x) \geq c\right\} \cap B_{3 R}\left(y_{0}\right) \backslash B_{2 R}\left(y_{0}\right)$, such that $\left\{\operatorname{Prj}\left(y_{1}\right), \operatorname{Prj}\left(y_{2}\right), \ldots, \operatorname{Prj}\left(y_{N}\right)\right\}$ are sparsely distributed on $\partial B_{2 R}\left(y_{0}\right)$ satisfying the following estimate:

$$
\mathrm{d}_{\mathrm{g}}\left(\operatorname{Prj}\left(y_{i}\right), \operatorname{Prj}\left(y_{j}\right)\right)>\frac{4 c_{2}}{c_{1}} r \quad \forall 1 \leq i<j \leq N .
$$

So by (3.9), we have

$$
\mathrm{d}_{\mathrm{g}}\left(\gamma_{s}^{y_{0} \operatorname{Prj}\left(y_{i}\right)}, \gamma_{t}^{y_{0} \operatorname{Prj}\left(y_{j}\right)}\right)>\frac{c_{1}}{2} \frac{4 c_{2}}{c_{1}} r=2 c_{2} r \quad \text { for any } s, t \in\left[\frac{1}{2}, 1\right] .
$$

By (3.13), we also have

$$
\bigcup_{t \in\left[\frac{1}{2}, \frac{2}{3}\right]} \operatorname{supp} \mu_{t}^{r, y_{i}} \subset \overline{\left(\left.\gamma_{t}^{y_{0} y_{i}}\right|_{t \in\left[\frac{1}{2}, \frac{2}{3}\right]}\right)_{c_{2} r}} \subset \overline{\left(\left.\gamma_{t}^{y_{0} \operatorname{Prj}\left(y_{i}\right)}\right|_{t \in\left[\frac{1}{2}, 1\right]}\right)_{c_{2} r}} .
$$

Combining (3.20) and (3.21), we know $\bigcup_{t \in\left[\frac{1}{2}, \frac{2}{3}\right]} \operatorname{supp} \mu_{t}^{r, y_{i}}, i=1, \ldots, N$ are disjoint.

Furthermore, consider the following set

$N\left(y_{i}, r\right):=\left\{\mathbf{t}=\left(t_{1}, t_{2}, \ldots\right): t_{i} \in\left[\frac{1}{2}, \frac{2}{3}\right], \operatorname{supp} \mu_{t_{1}}^{r, y_{i}}, \operatorname{supp} \mu_{t_{2}}^{r, y_{i}}, \ldots \subset B_{2 R}\left(y_{0}\right)\right.$ are disjoint $\}$.

It can be seen that $\max _{\mathbf{t} \in N\left(y_{i}, r\right)}|\mathbf{t}| \gtrsim \frac{1}{r}, i=1, \ldots, N$. 
In conclusion, we can find approximate $\frac{1}{r^{n}}$ measures whose supports are disjoint in $B_{2 R}\left(y_{0}\right)$. Combining with (3.12) and local finiteness of $\mathfrak{m}$ (c.f. Theorem 4.24 [30]) we obtain the following estimate

$$
r^{n} \min \left\{\epsilon_{r}\left(y_{0}\right), \epsilon_{r}\left(y_{1}\right), \ldots, \epsilon_{r}\left(y_{N}\right)\right\} \frac{1}{r^{n}}<C \mathfrak{m}\left(B_{2 R}\left(y_{0}\right)\right)<\infty
$$

where $C$ is independent of $c$. By the choice of $\left\{y_{1}, \ldots, y_{N}\right\}$, we know

$$
\min \left\{\epsilon_{r}\left(y_{1}\right), \ldots, \epsilon_{r}\left(y_{N}\right)\right\} \geq c .
$$

Letting $c \rightarrow \infty$, by (3.22) we get $\epsilon_{r}\left(y_{0}\right)<C \mathfrak{m}\left(B_{2 R}\left(y_{0}\right)\right)$, which is the contradiction. Therefore $\mathfrak{m}_{\mathrm{ac}} \neq 0$.

Step 2: $\mathfrak{m}_{\mathrm{s}}=0$ on $U$.

We will prove the assertion by contradiction. Assume that $\left.\mathfrak{m}\right|_{U}$ is not absolutely continuous w.r.t. $\mathrm{Vol}_{\mathrm{g}}$, then there exists a compact singular set $\mathrm{N} \subset U$ such that $\mathfrak{m}(\mathrm{N})=\mathfrak{m}_{\mathrm{s}}(\mathrm{N})>0$ and $\operatorname{Vol}_{\mathrm{g}}(\mathrm{N})=0$.

Since $\mathfrak{m}_{\mathrm{ac}} \neq 0$, there exists a bounded set $E_{L}$ with positive $\mathfrak{m}$-measure such that $\frac{\mathrm{d} \mathfrak{m}}{\mathrm{dVolg}}<L$ on $E_{L}$. Denote by $\left(\mu_{t}\right)$ the $L^{2}$-Wasserstein geodesic from $\mu_{0}:=\left.\frac{1}{\mathfrak{m}(\mathbb{N})} \mathfrak{m}\right|_{\mathbb{N}}$ to $\mu_{1}:=\left.\frac{1}{\mathfrak{m}\left(E_{L}\right)} \mathfrak{m}\right|_{E_{L}}$. By the choice of $E_{L}$, we know $\mu_{1} \ll V_{\mathrm{Vol}}$ with bounded density. By measure contraction property of $\left(\bar{U}, \mathrm{~d}_{\mathrm{g}}, \mathrm{Vol}_{\mathrm{g}}\right)$, we know $\mu_{t} \ll \operatorname{Vol}_{\mathrm{g}}$ for any $t>0$. In particular $\mu_{t}(\mathrm{~N})=0$, so there is a Borel set $A_{t} \subset \operatorname{supp} \mu_{t}$ such that $A_{t} \cap \mathrm{N}=\emptyset$ and $\mu_{t}\left(A_{t}\right)=1$. However, by Lemma 3.1 [27] again, we have $\mu_{t} \leq C_{1} \mathfrak{m}$ for some constant $C_{1}>0$. Next we will show the contradiction using the argument in [23] (c.f. Lemma 6.4 therein). Given $\epsilon>0$, we know

$$
A_{t} \subset \operatorname{supp} \mu_{t} \subset\left(\operatorname{supp} \mu_{0}\right)_{\epsilon}=(\mathrm{N})_{\epsilon}
$$

for $t$ small enough. Then

$$
\begin{aligned}
\mathfrak{m}(\mathrm{N}) & =\lim _{\epsilon \rightarrow 0} \mathfrak{m}\left((\mathrm{N})_{\epsilon}\right) \\
& \geq \varlimsup_{t \rightarrow 0} \mathfrak{m}\left(\operatorname{supp} \mu_{t}\right) \\
& \geq \varlimsup_{t \rightarrow 0} \mathfrak{m}\left(A_{t} \backslash \mathrm{N}\right)+\mathfrak{m}(\mathrm{N}) \\
& =\varlimsup_{t \rightarrow 0} \mathfrak{m}\left(A_{t}\right)+\mathfrak{m}(\mathrm{N}) \\
& \geq \varlimsup_{t \rightarrow 0} \frac{1}{C_{1}} \mu_{t}\left(A_{t}\right)+\mathfrak{m}(\mathrm{N}) \\
& \geq \frac{1}{C_{1}}+\mathfrak{m}(\mathrm{N})
\end{aligned}
$$

which is the contradiction.

Finally, since the choice of $U$ is arbitrary, we know $\mathfrak{m} \ll \operatorname{Vol}_{\mathrm{g}}$ on whole $M$.

\section{Part b):}


Given $x \in M$. For any $y \in B_{3 R}(x) \backslash \overline{B_{2 R}(x)}$, let us consider the $L^{2}$-Wasserstein geodesic $\left(\mu_{t}^{r}\right)_{t}$ from $\mu_{0}^{r}:=\left.\frac{1}{\mathfrak{m}\left(B_{r}(x)\right)} \mathfrak{m}\right|_{B_{r}(x)}$ to $\mu_{1}^{r}:=\delta_{y}$. By measure contraction property, we have the following (uniform) estimate

$$
\mathfrak{m}\left(\operatorname{supp} \mu_{t}^{r}\right) \gtrsim \mathfrak{m}\left(B_{r}(x)\right) \quad \forall t \in\left[0, \frac{2}{3}\right] .
$$

Combining the definition of $\epsilon_{r}$ (3.3) and (3.23), we get

$$
\mathfrak{m}\left(\operatorname{supp} \mu_{t}^{r}\right) \gtrsim r^{n} \epsilon_{r}(x) \quad \forall t \in\left[0, \frac{2}{3}\right] .
$$

As previously shown in Part 1), there exist (approximate) $\frac{1}{r^{n}}$ measures whose supports are disjoint. Combining with (3.24) we get

$$
\epsilon_{r}(x)=\left(r^{n} \epsilon_{r}(x)\right) \frac{1}{r^{n}} \lesssim \mathfrak{m}\left(B_{2 R}(x)\right)
$$

Since $\operatorname{MCP}(K, N)$ condition yields measure doubling property, $\mathfrak{m}\left(B_{2 R}(x)\right)$ is finite. Hence $\epsilon_{r} \in L^{\infty}\left(U, \mathrm{Vol}_{\mathrm{g}}\right)$ uniformly in $r$.

Letting $r \rightarrow 0$, by Lebesgue differentiation theorem there is $e^{-V} \in L_{\mathrm{loc}}^{\infty}\left(M, \operatorname{Vol}_{\mathrm{g}}\right)$ such that

$$
e^{-V(x)}=\lim _{r \rightarrow 0} \frac{\mathfrak{m}\left(B_{r}(x)\right)}{\operatorname{Vol}_{\mathrm{g}}\left(B_{r}(x)\right)}, \quad \operatorname{Vol}_{\mathrm{g}}-\text { a.e. } x .
$$

\section{Part c):}

For $x \in M$ and $0<r \ll 1$, we define

$$
\delta_{r}(x):=\frac{r^{N-n} \operatorname{Vol}_{\mathrm{g}}\left(B_{r}(x)\right)}{\mathfrak{m}\left(B_{r}(x)\right)} .
$$

Denote by $\left(\nu_{t}^{r}\right)_{t}$ the Wasserstein geodesic from $\nu_{0}^{r}:=\left.\frac{1}{\operatorname{Vol}_{\mathrm{g}}\left(B_{r}(x)\right)} \operatorname{Vol}_{\mathrm{g}}\right|_{B_{r}(x)}$ to $\nu_{1}^{r}:=$ $\delta_{y}$, with $0<r \ll R$. By measure contraction property (of compact Riemannian manifolds), we have

$$
\operatorname{Vol}_{\mathrm{g}}\left(\operatorname{supp} \nu_{t}^{r}\right) \gtrsim \operatorname{Vol}\left(B_{r}(x)\right) \quad \forall t \in\left[0, \frac{2}{3}\right] .
$$

Combining with (3.26), we obtain

$$
\operatorname{Vol}_{\mathrm{g}}\left(\operatorname{supp} \nu_{t}^{r}\right) \gtrsim r^{n-N} \delta_{r} \mathfrak{m}\left(B_{r}(x)\right) \quad \forall t \in\left[0, \frac{2}{3}\right] .
$$

By Bishop-Gromov inequality (c.f. Corollary 2.4 [31]), we know $\mathfrak{m}\left(B_{r}(x)\right) \gtrsim$ $\left(\frac{r}{R}\right)^{N} \mathfrak{m}\left(B_{2 R}(x)\right)$. Therefore (3.27) implies

$$
\operatorname{Vol}_{\mathrm{g}}\left(\operatorname{supp} \nu_{t}^{r}\right) \gtrsim \delta_{r} r^{n} \quad \forall t \in\left[0, \frac{2}{3}\right] \text {. }
$$


Similarly, we can find approximate $\frac{1}{r^{n}}$ measures whose supports are disjoint inside $B_{2 R}(x)$, then we obtain

$$
\delta_{r} \lesssim \operatorname{Vol}_{\mathrm{g}}\left(B_{2 R}(x)\right)
$$

Then we obtain the following $L_{\text {loc }}^{\infty}$ estimate

$$
\frac{r^{N-n} \operatorname{Vol}_{\mathrm{g}}\left(B_{r}(x)\right)}{\mathfrak{m}\left(B_{r}(x)\right)} \in L_{\mathrm{loc}}^{\infty} .
$$

If $N=n$, we have $\frac{\operatorname{Vol}_{\mathrm{g}}\left(B_{r}(x)\right)}{\mathfrak{m}\left(B_{r}(x)\right)} \in L_{\mathrm{loc}}^{\infty}$. Letting $r \rightarrow 0$, by Lebesgue differentiation theorem we know

$$
e^{V}=\lim _{r \rightarrow 0} \frac{\operatorname{Vol}_{\mathrm{g}}\left(B_{r}(x)\right)}{\mathfrak{m}\left(B_{r}(x)\right)} \in L_{\mathrm{loc}}^{\infty} .
$$

Combining with $e^{-V} \in L_{\text {loc }}^{\infty}$, we get $V \in L_{\text {loc }}^{\infty}$ (see also Proposition 3.5).

The following result has been proved in the Part 1) of the proof above (see also Lemma 6.4 [23]). For convenience of later applications, we extract it as a separate lemma.

Lemma 3.2. Let $\mu_{0}, \mu_{1}$ be two probability measures with compact support. Assume that $\mu_{1} \ll \operatorname{Vol}_{\mathrm{g}}$ and $\left(\mu_{t}\right) \subset \mathcal{W}_{2}(M, \mathrm{~g})$ is the unique $L^{2}$-Wasserstein geodesic connecting $\mu_{0}$ and $\mu_{1}$. If there exists a locally finite measure $\mathfrak{m}$ such that the density functions $\frac{\mathrm{d} \mu_{t}}{\mathrm{dm}}, t \in[0,1]$ are uniformly bounded. Then $\mu_{0} \ll \mathrm{Vol}_{\mathrm{g}}$.

\subsection{Measure rigidity: regularity of density, non-collapsing}

In the following two propositions, we will improve the regularity of density functions obtained in Proposition 3.1.

Proposition 3.3. Let $V: M \mapsto \mathbb{R} \cup\{+\infty\}$ be an extended-valued function on a complete Riemannian manifold $(M, \mathrm{~g})$, such that $\left(M, \mathrm{~d}_{\mathrm{g}}, e^{-V} \operatorname{Vol}_{\mathrm{g}}\right)$ is a $\mathrm{CD}(K, \infty)$ space. Then $V$ has a semi-convex, locally Lipschitz representative.

Proof. Denote $\mathfrak{m}:=e^{-V} \operatorname{Vol}_{\mathrm{g}}$. Let $\Omega \subset M$ be a convex, bounded open set such that points in $\Omega$ do not have cut-locus inside $\Omega$, and let $L>0$ be a constant such that the sub-level set $\{V \leq L\} \cap \Omega$ has positive $\mathfrak{m}$-measure (and hence positive Vol $_{\mathrm{g}}$ measure). Let $\mathcal{V}$ be a family of sets of bounded eccentricity defined in Lemma 3.4. Denote by $E_{L}$ the subset of $\{V \leq L\}$ which consists of density 1 points, i.e.

$$
E_{L}:=\left\{x: \lim _{U \in \mathcal{V} \rightarrow x} \frac{\operatorname{Vol}_{\mathrm{g}}(\{V \leq L\} \cap U)}{\operatorname{Vol}_{\mathrm{g}}(U)}=1\right\} .
$$

Firstly we will prove the following claim.

Step 1: For any $x \in E_{L}$, there is $r_{0}>0$ such that

$$
E_{L} \cap B_{r_{0}}(x)=\operatorname{Conv}\left(E_{L} \cap B_{r_{0}}(x)\right)^{\circ}
$$


where $\operatorname{Conv}\left(E_{L} \cap B_{r_{0}}(x)\right)^{\circ}$ is the interior points of the convex-hull of $E_{L} \cap B_{r_{0}}(x)$ in $\Omega$. In particular, $E_{L}$ is open and connected, $V$ is locally bounded from above.

Given $x \in E_{L}, v \in T_{x} M$ and $y:=\exp _{x}\left(\frac{1}{2} v\right)$. Let $\left(\mu_{t}^{\delta}\right)_{t \in[0,1]}$ denotes the $L^{2}$ Wasserstein geodesic from $\mu_{0}^{\delta}:=\left.\frac{1}{\operatorname{Volg}_{g}\left(E_{L} \cap A_{0}^{\delta}\right)} \operatorname{Vol}_{\mathrm{g}}\right|_{E_{L} \cap A_{0}^{\delta}}$ to $\mu_{1}^{\delta}:=\left.\frac{1}{\operatorname{Vol}_{\mathrm{g}}\left(A_{1}^{\delta}\right)} \operatorname{Vol}_{\mathrm{g}}\right|_{A_{1}^{\delta}}$, where $A_{0}^{\delta}=B_{\delta}(x), A_{1}^{\delta}=B_{\delta}\left(\exp _{x}(v)\right)$ are geodesic balls with radius $\delta>0$.

By direct computation (c.f. '(ii) $\Longrightarrow$ (i)' in the proof of Theorem 1.1 [33]), we have

$$
\operatorname{Ent}\left(\mu_{0}^{\delta} \mid \operatorname{Vol}_{\mathrm{g}}\right)=-\ln \operatorname{Vol}_{\mathrm{g}}\left(E_{L} \cap A_{0}^{\delta}\right)=-\ln c_{n}-n \ln \delta-\ln \frac{\operatorname{Vol}_{\mathrm{g}}\left(E_{L} \cap A_{0}^{\delta}\right)}{\operatorname{Vol}_{\mathrm{g}}\left(A_{0}^{\delta}\right)}+O\left(\delta^{2}\right),
$$

and

$$
\operatorname{Ent}\left(\mu_{1}^{\delta} \mid \operatorname{Vol}_{\mathrm{g}}\right)=-\ln \operatorname{Vol}_{\mathrm{g}}\left(A_{1}^{\delta}\right)=-\ln c_{n}-n \ln \delta+O\left(\delta^{2}\right)
$$

where $c_{n}:=\mathcal{L}^{n}\left(B_{1}\right)$ in $\mathbb{R}^{n}$.

Since $\Omega$ is compact, we may assume that the sectional curvature is bounded from above by $\kappa>0$. By Rauch's comparison theorem, for $\delta \ll|v| \ll 1$ we can find a set

$$
A_{\frac{1}{2}}^{\delta}=B_{\left(1+(\kappa+1 / n)|v|^{2} / 8\right) \delta}(y)
$$

such that $\gamma_{\frac{1}{2}} \in A_{\frac{1}{2}}^{\delta}$ for each minimizing geodesic $\gamma:[0,1] \mapsto M$ with $\gamma_{0} \in A_{0}^{\delta}, \gamma_{1} \in$ $A_{1}^{\delta}$. In particular, supp $\mu_{\frac{1}{2}}^{\delta} \subset A_{\frac{1}{2}}^{\delta}$. Moreover, by the asymptotic expansion formula in [33, Page 929, Proof of Theorem 1.1]

$$
\begin{aligned}
& \operatorname{Ent}\left(\mu_{\frac{1}{2}}^{\delta} \mid \operatorname{Vol}_{\mathrm{g}}\right) \\
\geq & -\ln \operatorname{Vol}_{\mathrm{g}}\left(A_{\frac{1}{2}}^{\delta}\right) \quad \text { (by Jensen's inequality) } \\
\geq & -\ln c_{n}-n \ln \delta-\frac{(n \kappa+1)|v|^{2}}{8}+O\left(\delta^{2}\right)+O\left(|v|^{4}\right) \quad \text { (c.f. page } 929 \text { in [33]). }
\end{aligned}
$$

Similarly, by the expansion formulas (3.32) and (3.33), for $k:=n \kappa+2$, we have

$$
\begin{aligned}
& \frac{1}{2} \operatorname{Ent}\left(\mu_{1}^{\delta} \mid \operatorname{Vol}_{\mathrm{g}}\right)+\frac{1}{2} \operatorname{Ent}\left(\mu_{0}^{\delta} \mid \operatorname{Vol}_{\mathrm{g}}\right)-\frac{k}{8} W^{2}\left(\mu_{0}^{\delta}, \mu_{1}^{\delta}\right) \\
= & -\ln c_{n}-n \ln \delta-\frac{1}{2} \ln \frac{\operatorname{Vol}_{\mathrm{g}}\left(E_{L} \cap B_{\delta}(x)\right)}{\operatorname{Vol}_{\mathrm{g}}\left(B_{\delta}(x)\right)}-\frac{k|v|^{2}}{8}+O(\delta) .
\end{aligned}
$$

Thus for $\delta$ and $|v|$ small enough, we have

$$
\operatorname{Ent}\left(\mu_{\frac{1}{2}}^{\delta} \mid \operatorname{Vol}_{\mathrm{g}}\right) \geq \frac{1}{2} \operatorname{Ent}\left(\mu_{1}^{\delta} \mid \operatorname{Vol}_{\mathrm{g}}\right)+\frac{1}{2} \operatorname{Ent}\left(\mu_{0}^{\delta} \mid \operatorname{Vol}_{\mathrm{g}}\right)-\frac{k}{8} W^{2}\left(\mu_{0}^{\delta}, \mu_{1}^{\delta}\right) .
$$

By the definition of $\operatorname{CD}(K, \infty)$, Ent $(\cdot \mid \mathfrak{m})$ is $K$-convex along $\left(\mu_{t}^{\delta}\right)$. Combining with the inequality (3.34) and the following formula

$$
\operatorname{Ent}(\cdot \mid \mathfrak{m})=\operatorname{Ent}\left(\cdot \mid \operatorname{Vol}_{\mathrm{g}}\right)+\int V \mathrm{~d}(\cdot),
$$


we obtain

$$
\begin{aligned}
& \int V \mathrm{~d} \mu_{\frac{1}{2}}^{\delta}-\frac{k-K}{8} W^{2}\left(\mu_{0}^{\delta}, \mu_{1}^{\delta}\right) \\
\leq & \frac{1}{2} \int V \mathrm{~d} \mu_{1}^{\delta}+\frac{1}{2} \int V \mathrm{~d} \mu_{0}^{\delta} .
\end{aligned}
$$

By replacing $V$ with $V+H$ for some locally $|K-k|$-convex function $H$ (and simultaneously replacing $\{V \leq L\}$ by $\{V+H \leq L\}$ ), we may assume $k=K$ without loss of generality.

By (3.35) and $E_{L} \subset\{V \leq L\}$, we have

$$
\int V \mathrm{~d} \mu_{\frac{1}{2}}^{\delta} \leq \frac{1}{2} \int V \mathrm{~d} \mu_{1}^{\delta}+\frac{1}{2} L
$$

If $y=\exp _{x}\left(\frac{1}{2} v\right)$ is a density 1 point of $\{V>L\}$, by(3.37) we get

$$
L<\varliminf_{\delta \rightarrow 0} \int V \mathrm{~d} \mu_{\frac{1}{2}}^{\delta} \leq \varliminf_{\delta \rightarrow 0} \frac{1}{2} \int V \mathrm{~d} \mu_{1}^{\delta}+\frac{1}{2} L,
$$

thus

$$
\gamma_{1}=\exp _{x}(v) \notin E_{L} .
$$

Let $r_{0}>0$ be small so that the geodesic ball $B_{r_{0}}(x)$ is geodesically convex. Assume by contradiction that $\operatorname{Vol}\left(\operatorname{Conv}\left(E_{L} \cap B_{r_{0}}(x)\right) \backslash E_{L}\right) \neq 0$. By Lebesgue density theorem, the density 1 points of $\{V>L\}$ in $B_{r_{0}}(x)$ are not negligible. By Fubini's theorem, there is $\gamma \subset B_{r_{0}}(x)$ such that $\gamma_{0}, \gamma_{1} \in E_{L}$ and $\gamma_{\frac{1}{2}}$ is a density 1 point of $\{V>L\}$, which contradicts to (3.38).

Notice that by Fubini's theorem we have $\operatorname{Vol}_{\mathrm{g}}\left(\partial \operatorname{Conv}\left(E_{L} \cap B_{r_{0}}(x)\right)\right)=0$. Hence

$$
E_{L} \cap B_{r_{0}}(x)=\operatorname{Conv}\left(E_{L} \cap B_{r_{0}}(x)\right)
$$

which is the thesis.

We define an extended real-valued function $\bar{V}: M \mapsto \mathbb{R} \cup\{ \pm \infty\}$ by

$$
\bar{V}(x):=\varliminf_{\nu \ni U \rightarrow x} \frac{1}{\operatorname{lol}_{\mathrm{g}}(U)} \int_{U} V \mathrm{dVol}_{\mathrm{g}}
$$

where $\mathcal{V}$ is the family of sets defined in Lemma 3.4.

Denote $V^{+}:=V \vee 0$ and $V^{-}:=V \wedge 0$. By the inequality $t \leq e^{t}$ on $[0,+\infty)$, we know $\left|V^{-}\right| \leq e^{-V}$ and $V^{-} \in L_{\mathrm{loc}}^{1}\left(\operatorname{Vol}_{\mathrm{g}}\right)$. Combining with the fact that $V^{+} \leq L$ on $E_{L}$, we get $V \in L^{1}\left(E_{L}, \operatorname{Vol}_{\mathrm{g}}\right)$. By Lebesgue differentiation theorem, we know $\bar{V}=V$ $\mathfrak{m}$-a.e. on $E_{L}$ and there is $M^{*} \subset E_{L}$ with full measure such that

$$
\lim _{\nu \ni U \rightarrow x} \frac{1}{\operatorname{Vol}_{\mathrm{g}}(U)} \int_{U} V \mathrm{dVol}_{\mathrm{g}}=\bar{V}(x) \in \mathbb{R} \quad \forall x \in M^{*} .
$$

Step 2: $\bar{V}$ is geodesically convex on $E_{L}$. Then from [18] (see also Corollary 3.10 [32]) we know $\bar{V}$ is locally Lipschitz on $M$. 
Let $\gamma \subset E_{L}$ be a geodesic with end points in $M^{*}$ and with small length, and let $\left(\mu_{t}^{\delta}\right)$ be the geodesic defined similarly as in Step 1. Similar to (3.35), we have

$$
\int V \mathrm{~d} \mu_{\frac{1}{2}}^{\delta} \leq \frac{1}{2} \int V \mathrm{~d} \mu_{1}^{\delta}+\frac{1}{2} \int V \mathrm{~d} \mu_{0}^{\delta}
$$

Letting $\delta \rightarrow 0$ in (3.39), we obtain

$$
\bar{V}\left(\gamma_{\frac{1}{2}}\right) \leq \frac{1}{2} \bar{V}\left(\gamma_{0}\right)+\frac{1}{2} \bar{V}\left(\gamma_{1}\right) \leq L
$$

Given $x \in E_{L}$, by Fubini's theorem we know there exists $S_{x} \subset\left\{v \in S^{1}\left(T_{x} M\right)\right\}$ with full $\mathcal{H}^{n-1}$-measure and a positive constant $\tau(x) \in(0,1]$, such that $\exp _{x}(t v) \in$ $M^{*}$ for all $v \in S_{x}$ and $\mathcal{L}^{1}$-a.e. $t \in(-\tau(x), \tau(x))$. Define a set of geodesic segments $\Gamma_{x}$ by

$$
\Gamma_{x}:=\left\{\left(\exp _{x}(t v)\right)_{t \in(-\tau(x), \tau(x))}: v \in S_{x}\right\} .
$$

Then for any $\gamma \in \Gamma_{x},(3.40)$ yields that $\bar{V}$ is convex on $\gamma \cap M^{*}$. In particular, $\bar{V}$ is Lipschitz on $\gamma \cap M^{*}$.

To prove the geodesical convexity of $\bar{V}$ on whole $E_{L}$, we just need to show the continuity of $\bar{V}$. Then by an approximation argument, we can see that $\bar{V}$ satisfies (3.40) on all geodesics. With this aim, we will prove the following claims.

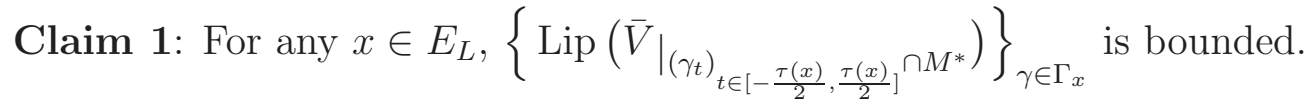

By (3.40) and the discussion thereafter, we know

$$
\bar{V}\left(\gamma_{0^{+}}\right):=\lim _{y \in \gamma \cap M^{*} \rightarrow x} \bar{V}(y) \in[\bar{V}(x), L]
$$

for any $\gamma \in \Gamma_{x}$. Thus by convexity we know $\bar{V}$ is $\frac{2(L-\bar{V}(x)+1)}{\tau}(x)$-Lipschitz on $\left\{\left(\gamma_{t}\right)_{t \in\left[-\frac{\tau(x)}{2}, \frac{\tau(x)}{2}\right]} \cap M^{*}: \gamma \in \Gamma_{x}\right\}$, which is the thesis.

We define a (possibly multi-valued) function $\bar{V}^{\prime}: E_{L} \mapsto[\bar{V}(x), L]$ by

$$
\bar{V}^{\prime}(x):=\left\{\bar{V}\left(\gamma_{0^{+}}\right): \gamma \in \Gamma_{x}\right\}
$$

For any $x \in M^{*}$, by (3.40) we know the value of $\bar{V}\left(\gamma_{0^{+}}\right)$is independent of the choice of the geodesic $\gamma \in \Gamma_{x}$ and $\bar{V}\left(\gamma_{0^{+}}\right)=\bar{V}(x)$, so $\bar{V}^{\prime}=V$ almost everywhere. Furthermore, if we can show that $\bar{V}^{\prime}$ is continuous, by definition we know $\bar{V}=\bar{V}^{\prime}$ on $E_{L}$. Therefore it suffices it to prove the following assertion.

Claim 2: $\bar{V}^{\prime}$ is single-valued and continuous on $E_{L}$.

Given $x \in E_{L}$. Assume by contradiction that $\bar{V}^{\prime}$ is not single-valued,

$$
-\infty<\bar{V}(x) \leq \bar{V}\left(\gamma_{0^{+}}^{1}\right)<\bar{V}\left(\gamma_{0^{+}}^{2}\right) \leq L
$$

for some different $\gamma^{1}, \gamma^{2} \in \Gamma_{x}$. By Fubini's theorem, we can find sequences $\left(x_{n}\right) \subset$ $\gamma^{1} \cap M^{*},\left(y_{n}\right) \subset \gamma^{2} \cap M^{*}$ such that $x_{n}, y_{n} \rightarrow x$, and $y_{n} \in \gamma^{x_{n}} \in \Gamma_{x_{n}}$. From (3.40), we can see that $\bar{V}^{\prime}\left(x_{n}\right) \rightarrow \bar{V}\left(\gamma_{0^{+}}^{1}\right), \bar{V}^{\prime}\left(y_{n}\right) \rightarrow \bar{V}\left(\gamma_{0^{+}}^{2}\right)$, and $\bar{V}^{\prime}$ is Lipschitz on $\gamma^{x_{n}} \cap M^{*}$. 
From (3.41) we also know $\left.\operatorname{Lip} \bar{V}^{\prime}\right|_{\gamma_{x_{n}}} \rightarrow+\infty$, which contradicts to the fact that $\bar{V}^{\prime} \leq L$ on $E_{L}$.

Finally, let $\left(z_{n}\right) \subset E_{L}$ be an arbitrary sequence with $z_{n} \rightarrow x$. By definition, we can find $z_{n}^{\prime} \in M^{*}$ such that $\mathrm{d}_{\mathrm{g}}\left(z_{n}^{\prime}, z_{n}\right)<\frac{1}{n}$ and $\left|\bar{V}\left(z_{n}^{\prime}\right)-\bar{V}^{\prime}\left(z_{n}\right)\right|<\frac{1}{n}$. By uniqueness of $\bar{V}^{\prime}(x)$ and Claim 1, we know $\bar{V}\left(z_{n}^{\prime}\right) \rightarrow \bar{V}^{\prime}(x)$. So $\bar{V}^{\prime}$ is continuous at $x$.

Lemma 3.4. We define a family of measurable sets $\mathcal{V}$ in the following way. We say that $U \in \mathcal{V}$ if there are $x, y \in M, \delta>0$, and a $L^{2}$-Wasserstein geodesic $\left(\mu_{t}^{\delta}\right)_{t \in[0,1]}$ from $\mu_{0}^{\delta}:=\left.\frac{1}{\operatorname{Vol}_{\mathrm{g}}\left(B_{\delta}(x)\right)} \operatorname{Vol}_{\mathrm{g}}\right|_{B_{\delta}(x)}$ to $\mu_{1}^{\delta}:=\left.\frac{1}{\operatorname{Vol}_{\mathrm{g}}\left(B_{\delta}(y)\right)} \operatorname{Vol}_{\mathrm{g}}\right|_{B_{\delta}(y)}$, such that $U=\operatorname{supp} \mu_{\frac{1}{2}}^{\delta}$.

Then $\mathcal{V}$ is a fine covering with bounded eccentricity. This means that every point $x \in M$ is covered by sets in $\mathcal{V}$ with arbitrarily small diameter, and there exists a constant $c>0$ such that each set $U \in \mathcal{V}$ is contained in a ball $B_{r}$ and $\operatorname{Vol}(U) \geq c \operatorname{Vol}\left(B_{r}\right)$.

Proof. Let $\left(T_{t}\right)$ be a family of maps which induce $\left(\mu_{t}^{\delta}\right)$, i.e. $\mu_{t}^{\delta}=\left(T_{t}\right)_{\sharp} \mu_{0}^{\delta}$. Denote the geodesic from $x$ to $y$ by $\gamma$. On one hand, by Rauch's comparison theorem, there exists a constant $C_{1}>0$ such that

$$
\mathrm{d}_{\mathrm{g}}\left(\gamma_{t}, T_{t}(z)\right) \leq C_{1}\left(\mathrm{~d}_{\mathrm{g}}\left(\gamma_{0}, z\right)+\mathrm{d}_{\mathrm{g}}\left(\gamma_{1}, T_{1}(z)\right)\right) \leq 2 C_{1} \delta
$$

for any $z \in \operatorname{supp} \mu_{0}^{\delta}=B_{\delta}(x)$. So we have

$$
\operatorname{supp} \mu_{t}^{\delta} \subset B_{2 C_{1} \delta}\left(\gamma_{t}\right) \text {. }
$$

On the other hand, Riemannian manifolds are locally CD spaces, hence

$$
\operatorname{Vol}_{\mathrm{g}}\left(\operatorname{supp} \mu_{t}^{\delta}\right) \gtrsim\left\{\operatorname{Vol}_{\mathrm{g}}\left(B_{\delta}(x)\right), \operatorname{Vol}_{\mathrm{g}}\left(B_{\delta}(y)\right)\right\} .
$$

Therefore by Bishop-Gromov inequality, the sets in $\mathcal{V}$ have bounded eccentricity. Furthermore, let $x=y \in M$, it can be seen that $U=B_{\delta}(x)$, so $\mathcal{V}$ is a fine covering of $M$.

Proposition 3.5. Let $V: M \mapsto \mathbb{R} \cup\{+\infty\}$ be an extended-valued function on a compact Riemannian manifold $(M, \mathrm{~g})$ with boundary, and $\mathfrak{m}=e^{-V} \operatorname{Vol}_{\mathrm{g}}$. If $\left(M, \mathrm{~d}_{\mathrm{g}}, \mathfrak{m}\right)$ satisfies $\operatorname{MCP}(K, N)$ for some $K \in \mathbb{R}$ and $N<\infty$. Then $V$ is locally bounded in the interior of $M$. In particular, $\left(M, \mathrm{~d}_{\mathrm{g}}, \mathfrak{m}\right)$ is infinitesimally Hilbertian.

Proof. We define the following family of functions with parameter $r \in(0,1)$, as in the proof of Proposition 3.1,

$$
\epsilon_{r}(x):=\frac{\mathfrak{m}\left(B_{r}(x)\right)}{\operatorname{Vol}_{\mathrm{g}}\left(B_{r}(x)\right)}
$$

Given $x \in M$ and $R>0$ with $B_{R}(x) \subset M$ and $\lim _{r \rightarrow 0} \epsilon_{r}(x)=e^{-V(x)}$. We define a family $\mathcal{V}$ of Borel sets in the following way. We say that $U \in \mathcal{V}$ if there exist 
$0<r \ll \frac{R}{2}, x_{0} \in B_{2 R}(x) \backslash B_{R}(x)$, and a $L^{2}$ - Wasserstein geodesic $\left(\mu_{t}\right)_{t \in[0,1]}$ with $\mu_{0}:=\delta_{x_{0}}$ and $\mu_{s}:=\left.\frac{1}{\mathfrak{m}\left(B_{r}(x)\right)} \mathfrak{m}\right|_{B_{r}(x)}$ for some $s \in\left[\frac{1}{3}, 1\right]$, such that $U=\operatorname{supp} \mu_{1}$. For the similar reason as Lemma 3.4, we know the sets in $\mathcal{V}$ is a covering of $H_{R}(x):=$ $B_{2 R}(x) \backslash B_{R}(x)$ with bounded eccentricity.

By Lebesgue differentiation theorem, there exists $H_{R}^{*}(x) \subset H_{R}(x)$ with full measure such that

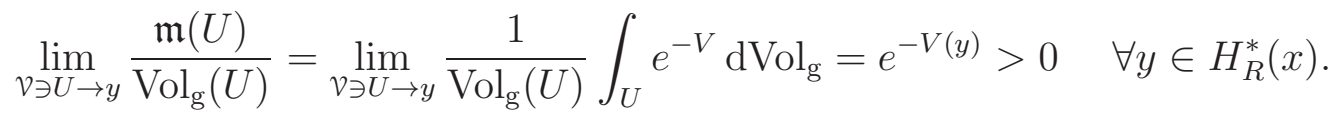

For any $y \in H_{R}^{*}(x)$ and $0<\delta \ll 1$. There is a $L^{2}$ - Wasserstein geodesic $\left(\mu_{t}\right)_{t \in[0,1]}$ with $\mu_{0}:=\delta_{x_{0}}$ and $\mu_{s}:=\left.\frac{1}{\mathfrak{m}\left(B_{r}(x)\right)} \mathfrak{m}\right|_{B_{r}(x)}$ for some $x_{0} \in B_{2 R}(x) \backslash B_{R}(x), s \in\left[\frac{1}{3}, 1\right]$ and $r=r(\delta)$, such that $U=\operatorname{supp} \mu_{1}$ and

$$
1-\delta<\left|\frac{\frac{\mathfrak{m}(U)}{\operatorname{Vol}(U)}}{e^{-V(y)}}\right|<1+\delta .
$$

By measure contraction property, there is a universal constant $C>0$ such that

$$
\epsilon_{r}(x) \operatorname{Vol}_{\mathrm{g}}\left(B_{r}(x)\right)=\mathfrak{m}\left(B_{r}(x)\right)>C \mathfrak{m}(U)>C(1-\delta) \operatorname{Vol}_{\mathrm{g}}(U) e^{-V(y)} .
$$

Dividing $r^{n}$ on both sides and letting $r \rightarrow 0$, we get $e^{-V(x)} \gtrsim e^{-V(y)}$. Recall that $H_{R}^{*}(x)$ has full measure in $H_{R}(x)$, we have the following weak mean-value property

$$
e^{-V(x)} \gtrsim \mathfrak{m}\left(B_{R}(x)\right)>0 .
$$

Combining with Proposition 3.1, we know $V \in L_{\text {loc }}^{\infty}$.

Next we will prove that there is no non-trivial measure other than the volume measure such that a $n$-dimensional Riemannian manifold satisfies $\mathrm{CD}(K, n)$ condition. We remark that this result can also be obtained by combining KapovitchKetterer's recent result [22, Corollary 1.2], and Cavalletti-Mondino's result [10, Corollary 8.3] about measure rigidity on Alexandrov spaces (see also [21, Theorem 1.4] for a more recent proof).

Theorem 3.6 (Measure Rigidity: non-collapsed spaces). Let ( $M, \mathrm{~g})$ be a $n$-dimensional Riemannian manifold. Assume there exists a measure $\mathfrak{m}^{*}$ with full support such that $\left(M, \mathrm{~d}_{\mathrm{g}}, \mathfrak{m}^{*}\right)$ is $\mathrm{CD}(K, n)$ for some $K \in \mathbb{R}$. Then there exists a constant $c>0$ such that $\mathfrak{m}^{*}=c \mathrm{Vol}_{\mathrm{g}}$.

Proof. For any $x \in M$, we can find a small convex neighborhood $U$ of it, such that $\left(\bar{U}, \mathrm{~d}_{g}, \mathfrak{m}^{*}\right)$ is still $\operatorname{CD}(K, n)$ and $\left(\bar{U}, \mathrm{~d}_{\mathrm{g}}, \operatorname{Vol}_{g}\right)$ is a $\operatorname{CD}(k, n)$ for some $k \in \mathbb{R}$. So without loss of generality, we may assume that any point in $M$ has no cut-locus and $\left(M, \mathrm{~d}_{\mathrm{g}}, \mathrm{Vol}_{\mathrm{g}}\right)$ is $\mathrm{CD}(k, n)$ for some $k$. By Proposition 3.1 and Proposition 3.3 we know there exists a positive continuous function $\varphi$ such that $\mathfrak{m}^{*}=\varphi^{n} \operatorname{Vol}_{g}$. Hence we just need to prove that $\varphi$ is a constant. 
Given two points $x, y \in M$. Let $\gamma$ be a geodesic from $x$ to $y$. Let $\mathfrak{m}=\mathfrak{m}^{*}=$ $\varphi^{n} \mathrm{Vol}_{\mathrm{g}}$ and $\mathfrak{m}=\mathrm{Vol}_{\mathrm{g}}$ respectively. By Brunn-Minkowski inequality on $\operatorname{CD}(K, n)$ spaces (c.f. [31, Proposition 2.1] ) and Rauch's comparison theorem, there is $C>0$ such that

$$
\mathfrak{m}\left(B_{\frac{\sigma}{2}\left(1+C \mathrm{~d}_{\mathfrak{g}}^{2}(x, y)\right)}\left(\gamma_{\frac{1}{2}}\right)\right)^{\frac{1}{n}} \geq \tau_{K, n}^{\left(\frac{1}{2}\right)}(\Theta) \mathfrak{m}\left(B_{\sigma}(x)\right)^{\frac{1}{n}}
$$

where $0<\sigma \ll 1$ and $\left|\Theta-\mathrm{d}_{\mathrm{g}}(x, y)\right| \leq \sigma$.

We define $\mathcal{J}_{\mathfrak{m}}(x)$ by

$$
\mathcal{J}_{\mathfrak{m}}(x):=\lim _{r \rightarrow 0}\left(\frac{\mathfrak{m}\left(B_{r}(x)\right)}{r^{n}}\right)^{\frac{1}{n}}
$$

Dividing $\frac{\sigma}{2}\left(1+C \mathrm{~d}_{\mathrm{g}}^{2}(x, y)\right)$ on both sides of (3.42) and letting $\sigma \rightarrow 0$, we obtain

$$
\mathcal{\partial}_{\mathfrak{m}}\left(\gamma_{\frac{1}{2}}\right) \geq \frac{2}{1+C \mathrm{~d}_{\mathfrak{g}}^{2}(x, y)} \tau_{K, n}^{\left(\frac{1}{2}\right)}\left(\mathrm{d}_{\mathfrak{g}}(x, y)\right) \mathcal{J}_{\mathfrak{m}}(x) .
$$

When $\mathrm{d}_{\mathrm{g}}(x, y)$ is small, by Taylor expansion of $\tau_{K, n}^{\left(\frac{1}{2}\right)}(\theta)$ we obtain

$$
\partial_{\mathfrak{m}}\left(\gamma_{\frac{1}{2}}\right) \geq \frac{1+O\left(\mathrm{~d}_{\mathrm{g}}^{2}(x, y)\right)}{1+C \mathrm{~d}_{\mathrm{g}}^{2}(x, y)} \partial_{\mathfrak{m}}(x) .
$$

For any $N>0$, we divide $\gamma$ equally into $N$ parts. Repeating the argument above on each interval with length $\frac{1}{N} \mathrm{~d}_{\mathrm{g}}(x, y)$ we get

$$
\mathcal{J}_{\mathfrak{m}}\left(\gamma_{\frac{i+1}{N}}\right) \geq\left(1+o\left(\frac{1}{N}\right)\right) \mathcal{J}_{\mathfrak{m}}\left(\gamma_{\frac{i}{N}}\right) \quad i=0, \ldots, N-1
$$

Therefore

$$
\mathcal{\partial}_{\mathfrak{m}}(y) \geq\left(1+o\left(\frac{1}{N}\right)\right)^{N} \mathcal{\partial}_{\mathfrak{m}}(x) .
$$

Letting $N \rightarrow \infty$, we obtain $\mathcal{J}_{\mathfrak{m}}(y) \geq \mathcal{J}_{\mathfrak{m}}(x)$. By symmetry, we can also prove $\mathcal{J}_{\mathfrak{m}}(y) \leq \mathcal{J}_{\mathfrak{m}}(x)$, hence $\mathcal{J}_{\mathfrak{m}}(y)=\mathcal{J}_{\mathfrak{m}}(x)$. So $\mathcal{J}_{\mathfrak{m}}$ is a constant for both $\mathfrak{m}=\operatorname{Vol}_{\mathrm{g}}$ and $\mathfrak{m}=\mathfrak{m}^{*}$. By Proposition 3.5 we know $\varphi$ is continuous, so we also have

$$
\mathcal{J}_{\mathfrak{m}^{*}}=\varphi \mathcal{J}_{\mathrm{Volg}_{\mathrm{g}}} .
$$

Therefore $\varphi$ is a constant.

\subsection{Measure rigidity: geodesical convexity}

In the last theorem, we study the $\mathrm{CD}(K, \infty)$ condition on manifolds with Lipschitz boundary. Without loss of generality, we may restrict our study on an open set $\Omega \subset M$ with Lipschitz boundary, which means that the boundary $\partial \Omega$ can be written locally as the graph of a Lipschitz continuous function on $\mathbb{R}^{n-1}$. 
As we mentioned in the introduction, no matter how smooth the boundary is, we cannot definitely predict that the geodesics are $C^{2}$. Consider the complement of a disc in the Euclidean plane. A geodesic fails to have an acceleration only at those points which we call switch points, where the geodesic switches from a boundary segment to an interior segment or vice-versa. In addition, besides the switch points, boundary segments, and interior segments, one other kind of point is possible, an accumulation point of switch points, which we call intermittent point. It is not difficult to construct a geodesic whose intermittent points form a Cantor set with positive measure. Unfortunately, it is uncertain which assumptions on the boundary guarantee finite switching behavior. One known result (c.f. [2]) is that domains in Euclidean plane with analytic boundary have no intermittent point. However, thanks to a theorem proved by Alexander, Berg and Bishop (Theorem 1 [2]), these intermittent points will not bring us too much trouble in our problem.

Theorem 3.7 (Measure rigidity: $\mathrm{CD}(K, \infty)$ condition). Let $(M, \mathrm{~g})$ be a complete Riemannian manifold, $\Omega \subset M$ be an open set with Lipschitz boundary. Let $\mathrm{d}_{\Omega}$ be the intrinsic distance induced by the Riemannian distance $\mathrm{d}_{\mathrm{g}}$ on $\bar{\Omega}$, and $\mathfrak{m}$ be a reference measure with supp $\mathfrak{m}=\bar{\Omega}$. Assume that $\partial \Omega$ is $C^{2}$ out of a $\mathcal{H}^{n-1}$-negligible set, and $\left(\bar{\Omega}, \mathrm{d}_{\Omega}, \mathfrak{m}\right)$ satisfies the $\operatorname{CD}(K, \infty)$ condition, then we have the following rigidity results.

1) $\bar{\Omega}$ is g-geodesically convex, this is to say, any shortest path in $\left(\bar{\Omega}, \mathrm{d}_{\Omega}\right)$ is a (unparameterized) geodesic (segment) in $(M, \mathrm{~g})$;

2) $\mathfrak{m}(\partial \Omega)=0$ and $\mathfrak{m}=e^{-V} \operatorname{Vol}_{\mathrm{g}}$ for some semi-convex, locally Lipschitz function $V$ on $\Omega$;

3) $\left(\bar{\Omega}, \mathrm{d}_{\Omega}, \mathfrak{m}\right)$ is a $\operatorname{RCD}(K, \infty)$ space.

In particular, $\left(\bar{\Omega}, \mathrm{d}_{\Omega}, \operatorname{Vol}_{\mathrm{g}}\right)$ is $\mathrm{CD}(K, \infty)$ if and only if $\bar{\Omega}$ is g-geodesically convex and Ricci $\geq K$ on $\Omega$.

Proof. Since all the assertions are local, without loss of generality, we may assume that $\bar{\Omega}$ is compact and points in $\bar{\Omega}$ do not have cut-locus inside $\bar{\Omega}$.

First of all, by Proposition 3.1 and Proposition 3.3 we know $\left.\mathfrak{m}\right|_{\Omega} \ll \operatorname{Vol}_{\text {g }}$, $\left.\operatorname{Vol}_{\mathrm{g}} \ll \mathfrak{m}\right|_{\Omega}$ and $\mathfrak{m}=e^{-V} \operatorname{Vol}_{\mathrm{g}}$ for some semi-convex, locally Lipschitz function $V$. In particular, we have

$$
\frac{\mathrm{dVol}_{\mathrm{g}}}{\mathrm{d} \mathfrak{m}} \in L^{\infty}(\Omega, \mathfrak{m}), \quad \frac{\mathrm{d} \mathfrak{m}}{\mathrm{dVol}_{\mathrm{g}}} \in L^{\infty}\left(\Omega, \operatorname{Vol}_{\mathrm{g}}\right) .
$$

Given $x, y \in \Omega$ and a parameter $\epsilon>0$ such that $B_{\epsilon}(x), B_{\epsilon}(y) \subset \Omega$. Firstly, consider the $L^{1}$-optimal transportation on $\left(\bar{\Omega}, \mathrm{d}_{\Omega}\right)$ between $\mu_{0}^{\epsilon}:=\left.\frac{1}{\mathfrak{m}\left(B_{\epsilon}(x)\right)} \mathfrak{m}\right|_{B_{\epsilon}(x)}$ and $\mu_{1}^{\epsilon}:=\left.\frac{1}{\mathfrak{m}\left(B_{\epsilon}(y)\right)} \mathfrak{m}\right|_{B_{\epsilon}(y)}$. Let $\left(\mu_{t}^{\epsilon}\right)_{t}$ be a geodesic from $\mu_{0}^{\epsilon}$ to $\mu_{1}^{\epsilon}$ in $L^{1}$-Wasserstein space $\mathcal{W}_{1}\left(\bar{\Omega}, \mathrm{d}_{\Omega}\right)$. Denote by $\Pi^{\epsilon}$ its lifting in $\mathcal{P}\left(\operatorname{Geod}\left(\bar{\Omega}, \mathrm{d}_{\Omega}\right)\right)$ satisfying $\left(e_{t}\right)_{\sharp} \Pi^{\epsilon}=\mu_{t}^{\epsilon}$. By $L^{1}$-optimal transport theory, there exists a Kantorovich potential $\varphi$ associated with such optimal transportation, which is a 1-Lipschitz function. Let $\Gamma^{\varphi}$ be the subset of 
$\mathrm{C}\left([0,1] ;\left(\bar{\Omega}, \mathrm{d}_{\Omega}\right)\right)$ containing all the (parametrized) trajectories of the gradient flow of $\varphi$. It is known that $\Pi^{\epsilon}\left(\Gamma^{\varphi}\right)=1$.

For $0<\delta \ll \frac{1}{2}$ small enough, $\left(\mu_{t}^{\epsilon}\right)_{t \in[0, \delta]}$ and $\left(\mu_{t}^{\epsilon}\right)_{t \in[1-\delta, 1]}$ are also $L^{1}$-Wasserstein geodesics (segments) in $\mathcal{W}_{1}\left(\bar{\Omega}, \mathrm{d}_{\mathrm{g}}\right)$. By needle decomposition via $L^{1}$-optimal transport (c.f. Theorem 3.8, Theorem $5.1[11]$ ), there is $\Gamma \subset \Gamma^{\varphi}$ such that $\Pi^{\epsilon}\left(\Gamma^{\varphi} \backslash \Gamma\right)=0$ and $\left(\gamma_{t}\right)_{t \in[0, \delta] \cup[1-\delta, 1]}, \gamma \in \Gamma$ are pairwisely disjoint. In addition, the measure $\left.\operatorname{Vol}_{\mathrm{g}}\right|_{B_{\epsilon}(x)}$ (and similarly $\left.\operatorname{Vol}_{\mathrm{g}}\right|_{B_{\epsilon}(y)}$ ) has a decomposition

$$
\left.\operatorname{Vol}_{\mathrm{g}}\right|_{B_{\epsilon}(x)}=\int_{\mathfrak{Q}} \mathfrak{m}_{q} \mathrm{~d} \mathfrak{q}
$$

where $\mathfrak{Q}$ can be represented locally as a level set of $\varphi$, and $\left(\mathfrak{m}_{q}\right)_{q}$ support on disjoint geodesic segments $\left(X_{q}\right)_{q}$. Furthermore, $\mathfrak{m}_{q} \ll \mathcal{H}^{1}$ and $h_{q}=\frac{\mathrm{dm}_{q}}{\mathrm{~d}^{1}}$ is a $\operatorname{CD}(k, n)$ density for q-a.e. q., this means that $\left(X_{q},|\cdot|, \mathfrak{m}_{q}\right)$ satisfies $\operatorname{BE}(k, n)$ condition in the sense of Bakry-Émery. Thus for any $q, h_{q}$ is Lipschitz and it can not vanish at the interior points of $X_{q}$.

Next we will construct a $L^{2}$-optimal transportation based on $\Pi^{\epsilon}$ and $\Gamma$.

Denote the level set $\{\varphi=T\}$ by $\varphi_{T}$. For any $z \in B_{\epsilon}(x)$, there exist a $\gamma^{z} \in \Gamma$ such that $z \in \gamma^{z}$, and a unique $T_{z}:=\varphi(z)$ such that $z=\varphi_{T_{z}} \cap \gamma^{z}$. In addition, by Fubini's theorem, there exists $T_{0}$ such that $B^{*}:=\left\{z: \gamma^{z} \cap \varphi_{T_{z}-T_{0}} \in B_{\epsilon}(y)\right\} \cap B_{\epsilon}(x)$ has positive Vol $_{\mathrm{g}}$-volume. It can be seen that $\mathrm{Cpl}:=\left\{\left(z_{1}, z_{2}\right): z_{1} \in B^{*}, z_{2} \in \gamma^{z_{1}} \cap \varphi_{T_{z_{1}}-T_{0}}\right\} \subset$ $B_{\epsilon}(x) \times B_{\epsilon}(y)$ is still a $L^{1}$-optimal transport coupling. Furthermore, we have

$$
\begin{aligned}
& \left(\varphi\left(y_{1}\right)-\varphi\left(y_{0}\right)\right)\left(\varphi\left(x_{1}\right)-\varphi\left(x_{0}\right)\right) \\
= & \left(\left(\varphi\left(x_{1}\right)-T_{0}\right)-\left(\varphi\left(x_{0}\right)-T_{0}\right)\right)\left(\varphi\left(x_{1}\right)-\varphi\left(x_{0}\right)\right) \\
= & \left(\varphi\left(x_{1}\right)-\varphi\left(x_{0}\right)\right)^{2} \geq 0
\end{aligned}
$$

for any $\left(x_{0}, y_{0}\right),\left(x_{1}, y_{1}\right) \in \mathrm{Cpl}$. By Lemma 4.6 in [9], we know Cpl is $\mathrm{d}_{\Omega^{2}}^{2}$ cyclically monotone, so that it is also a $L^{2}$-optimal transport coupling (c.f. Theorem 2.13 [4]). From the construction of $\mathrm{Cpl}$ we know $(\mathrm{Cpl})_{z_{1}}=B^{*}$ has positive $\mathfrak{m}$-measure, and by measure decomposition (3.44) and the regularity of $\operatorname{CD}(k, n)$ densities, we also have $\mathfrak{m}\left((\mathrm{Cpl})_{z_{2}}\right)>0$. Then by renormalization we obtain a curve, still denote it by $\left(\mu_{t}^{\epsilon}\right)$, which is a $L^{1}$-Wasserstein geodesic, as well as a $L^{2}$-Wasserstein geodesic. From the construction above, we can see that both $\mu_{0}^{\epsilon}, \mu_{1}^{\epsilon}$ have bounded $\mathfrak{m}$-densities.

To prove the geodesical convexity of $\bar{\Omega}$, we just need to show that $\Pi^{\epsilon}\left(\operatorname{Geod}\left(\bar{\Omega}, \mathrm{d}_{\Omega}\right) \backslash\right.$ $\operatorname{Geod}(M, \mathrm{~g}))=0$, then letting $\epsilon \rightarrow 0$ we know that $x$ and $y$ are connected by a geodesic in $(M, \mathrm{~g})$.

Let $\mathrm{R}$ be the set of $C^{2}$-regular points of $\partial \Omega$. By assumption, $\mathcal{H}^{n-1}(\partial \Omega \backslash \mathrm{R})=0$. It can be seen that $\operatorname{Geod}\left(\bar{\Omega}, \mathrm{d}_{\Omega}\right)$ has a decomposition $\operatorname{Geod}\left(\bar{\Omega}, \mathrm{d}_{\Omega}\right)=\Gamma^{1} \cup \Gamma^{2} \cup \Gamma^{3}$, where
a) $\Gamma^{1}=\left\{\gamma: \mathcal{H}^{1}(\gamma \cap \mathrm{R})>0\right\}$
b) $\Gamma^{2}=\left\{\gamma: \mathcal{H}^{1}(\gamma \cap \mathrm{R})=0, \gamma \cap \partial \Omega \subset \mathrm{R}\right\}$; 
c) $\Gamma^{3}=\left\{\gamma: \mathcal{H}^{1}(\gamma \cap \mathrm{R})=0, \gamma \cap \partial \Omega \backslash \mathrm{R} \neq \varnothing\right\}$.

We will prove $\Pi^{\epsilon}\left(\Gamma^{i}\right)=0$ for $i=1,3$, and $\Gamma^{2} \subset \operatorname{Geod}(M, \mathrm{~g})$ in the following three steps.

Step 1: $\Pi^{\epsilon}\left(\Gamma^{1}\right)=0$.

By Proposition 3.1 and Proposition 3.3, there exists a locally Lipschitz and semiconvex function $V$ such that

$$
\mathfrak{m}=\left.e^{-V} \operatorname{Vol}_{\mathfrak{g}}\right|_{\Omega}+\left.\mathfrak{m}\right|_{\partial \Omega \backslash \mathrm{R}}+\left.\mathfrak{m}\right|_{\mathrm{R}}
$$

In particular, $\left.\mathfrak{m}\right|_{\Omega}<\left.C_{0} \operatorname{Vol}_{\mathrm{g}}\right|_{\Omega}$ for some $C_{0}>0$.

Claim: $\mathfrak{m}(\mathrm{R})=0$, therefore $\mathfrak{m}=\left.e^{-V} \operatorname{Vol}_{\mathfrak{g}}\right|_{\Omega}+\left.\mathfrak{m}\right|_{\partial \Omega \backslash \mathrm{R}}$.

Assume that $\partial \Omega$ is locally represented as the graph of a bi-Lipschitz function $\phi$ on $U \subset \mathbb{R}^{n-1}$, and

$$
\frac{1}{L}|a b|<\mathrm{d}_{\mathrm{g}}(x, y)<L|a b| \quad \forall a, b \in U, x=(a, \phi(a)), y=(b, \phi(b))
$$

for some $L>1$. For any $a \in U$ with $(a, \phi(a)) \in \mathrm{R}$, there is a unique tangent plane $\mathrm{d} \phi(a)$ at this point and

$$
\lim _{r \rightarrow 0} \sup _{b \in U,|b-a|<r} \frac{|\phi(b)-\phi(a)-\mathrm{d} \phi(a)(b-a)|}{|b-a|}=0 .
$$

In particular, there exists a unique inward (unit) normal vector field $\mathrm{R} \ni x \mapsto \mathrm{N}_{x}$ such that $\mathrm{N}_{x} \perp \mathrm{d} \phi(x)$. Furthermore, for any $x \in \mathrm{R}$, there is $\delta(x)>0$ such that for any $r \leq \delta(x)$, all $\mathrm{d}_{\Omega}$-geodesics from $B_{r}\left(\exp _{x}\left(3 r \mathrm{~N}_{\mathrm{x}}\right)\right)$ to $x$ are g-geodesics.

Assume by contradiction that $\mathfrak{m}(\mathrm{R}) \neq 0$. By Lusin's theorem there exists $\mathrm{R}^{*} \subset \mathrm{R}$ with $\mathfrak{m}\left(\mathrm{R}^{*}\right)>0$ and a constant $r_{0}>0$, such that $\delta(x) \geq r_{0}$ and the map $x \rightarrow \mathrm{N}_{x}$ is continuous on $\mathrm{R}^{*}$.

Let $\left.x \in \mathrm{R}^{*} \cap \operatorname{supp} \mathfrak{m}\right|_{\mathrm{R}^{*}}$. There is a neighbourhood $U_{x} \subset \partial \Omega$ of $x$, such that all the $\mathrm{d}_{\Omega}$-geodesics connecting $U_{x} \cap \mathrm{R}^{*}$ and $B_{\frac{r_{0}}{2}}\left(\exp _{x}\left(3 r_{0} \mathrm{~N}_{\mathrm{x}}\right)\right)$ are g-geodesics. By Lemma 3.2, we get the contradiction. Therefore $\mathfrak{m}(\mathrm{R})=0$ and we prove the claim.

Assume by contradiction that $\Pi^{\epsilon}\left(\Gamma^{1}\right)>0$. By Fubini's theorem we know

$$
\left(\Pi^{\epsilon} \times L^{1}\right)\left(\left\{(\gamma, t): \gamma \in \Gamma_{1}, t \in[0,1], \gamma_{t} \in \mathrm{R}\right\}\right)>0,
$$

and there is $t_{0} \in[0,1]$ such that

$$
\Pi^{\epsilon}\left(\left\{\gamma: \gamma \in \Gamma^{1}, \gamma_{t_{0}} \in \mathrm{R}\right\}\right)>0
$$

so $\mu_{t_{0}}^{\epsilon}(\mathrm{R})>0$, which contradicts to the facts that $\mu_{t_{0}}^{\epsilon} \ll \mathfrak{m}$ and $\left.\mathfrak{m}\right|_{\mathrm{R}}=0$. Therefore $\Pi^{\epsilon}\left(\Gamma^{1}\right)=0$. 
Step 2: $\Gamma^{2} \subset \operatorname{Geod}(M, \mathrm{~g})$.

Let $\gamma \in \Gamma^{2}$. For any $t \in[0,1]$ with $\gamma_{t} \in \Omega$, it is known that $\ddot{\gamma}_{t}=0$. For any $t \in[0,1]$ with $\gamma_{t} \in \partial \Omega$. From the definition of $\Gamma^{2}$ we know $\gamma_{t} \in \mathrm{R}$, so $\dot{\gamma}_{t}$ exists.

Since $\gamma \cap \partial \Omega$ is closed and $\mathcal{H}^{1}$-negligible, any $\gamma_{t} \in \mathrm{R}$ is either an isolate point or an intermittent point. For isolate points, by elementary calculus we know $\ddot{\gamma}_{t}$ exists and equals to 0 . For intermittent points, by Theorem 1 in [2] we also know $\ddot{\gamma}_{t}$ exists and equals to 0 .

So $\ddot{\gamma}_{t} \equiv 0$ and $\gamma \in \operatorname{Geod}(M, \mathrm{~g})$ which is the thesis.

Step 3: $\Pi^{\epsilon}\left(\Gamma^{3}\right)=0$.

By definition and $\Gamma^{2} \subset \operatorname{Geod}(M, \mathrm{~g})$ which is proved in the last step, for any $\gamma \in \Pi^{\epsilon}\left(\Gamma^{3}\right)$, there is a point $b(\gamma) \in \gamma \cap \partial \Omega \backslash R$ such that the segment from $\gamma_{0}$ to $b(\gamma)$ is a Riemannian geodesic (segment).

Recall the decomposition $\left.\operatorname{Vol}_{g}\right|_{B_{\epsilon}(x)}=\int_{\mathfrak{Q}} \mathfrak{m}_{q} \mathrm{~d} \mathfrak{q}$ in (3.44) and keep the notations thereafter. For any $z \in B_{\epsilon}(x)$, there a longest $\gamma^{z} \in \Gamma^{3}$ such that $z \in \gamma^{z}$, and a unique $T_{z}=\varphi(z) \in \mathbb{R}$ such that $z=\varphi_{T_{z}}$. In addition, for such $\gamma^{z}$, there is a unique $q^{z} \in \mathfrak{Q}$ such that $\operatorname{supp} \mathfrak{m}_{q^{z}} \subset \gamma^{z}$.

Assume by contradiction that $\Pi^{\epsilon}\left(\Gamma^{3}\right)>0$. Denote $\mathfrak{Q}^{T}$ by

$$
\mathfrak{Q}^{T}:=\left\{q^{z}: z \in B_{\epsilon}(x), \gamma^{z} \in \Gamma^{3}, \varphi\left(\gamma_{0}^{z}\right)-\varphi(z)>\frac{\epsilon}{8} \text { and } \varphi_{T_{z}-T} \cap \gamma^{z}=b\left(\gamma^{z}\right)\right\} .
$$

Then by Fubini's theorem, there is $T_{1}>0$ such that $\mathfrak{q}\left(\mathfrak{Q}^{T_{1}}\right)>0$.

For $\sigma \in(0, \delta)$, we define couplings $\mathrm{Cpl}_{\sigma}^{1,2}, \mathrm{Cpl}_{\sigma}^{1,3} \subset M \times M$ by

$$
\mathrm{Cpl}_{\sigma}^{1,2}:=\left\{\left(z_{1}, z_{2}\right): z_{1} \in \gamma \cap \varphi_{T_{b(\gamma)}+T_{1}-s \sigma}, z_{2} \in \gamma \cap \varphi_{T_{b(\gamma)}+(1-s) \sigma}, \gamma \in \Gamma^{3}, s \in[0,1]\right\}
$$

and

$$
\mathrm{Cpl}_{\sigma}^{1,3}:=\left\{\left(z_{1}, z_{3}\right): z_{1} \in \gamma \cap \varphi_{T_{b(\gamma)}+T_{1}-s \sigma}, z_{3} \in \gamma \cap \varphi_{T_{a(\gamma)}-s \sigma-T_{0}}, \gamma \in \Gamma^{3}, s \in[0,1]\right\} .
$$

By the choice of $T_{1}$ and the regularity of conditional measures $\mathfrak{m}_{q}$, we can see that $\mathfrak{m}\left(\left(\mathrm{Cpl}_{\sigma}^{1,2}\right)_{z_{1}}\right), \mathfrak{m}\left(\left(\mathrm{Cpl}_{\sigma}^{1,3}\right)_{z_{3}}\right)>0$. More precisely, we have

$$
\operatorname{Vol}\left(\left(\mathrm{Cpl}_{\sigma}^{1,2}\right)_{z_{1}}\right) \geq \int_{\mathfrak{Q}^{T_{1}}} \mathfrak{m}_{q}\left(\left\{\varphi_{T_{b(\gamma)}+T_{1}-s \sigma} \cap \gamma: \gamma \in \Gamma^{3}, s \in[0,1]\right\}\right) \mathrm{d} \mathfrak{q}=O(\sigma)>0
$$

and similarly

$$
\operatorname{Vol}\left(\left(\mathrm{Cpl}_{\sigma}^{1,2}\right)_{z_{1}}\right) \gtrsim \sigma
$$

From the construction, we can see that these couplings are $\mathrm{d}_{\Omega}$-cyclically monotone, as well as $\mathrm{d}_{\Omega}^{2}$-cyclically monotone (c.f. (3.45) and Lemma 4.6 [9]). Therefore, they are both $L^{1}$-optimal and $L^{2}$-optimal (c.f. Theorem 2.13 [4]). By renormalization and reparameterization, we can find a Wasserstein geodesic $\left(\nu_{t}^{\sigma}\right)$ in $\mathcal{W}_{2}\left(\bar{\Omega}, \mathrm{d}_{\Omega}\right) \cap \mathcal{W}_{1}\left(\bar{\Omega}, \mathrm{d}_{\Omega}\right)$, such that $\mathrm{Cpl}_{\sigma}^{1,2}$ is the optimal coupling for $\left(\nu_{0}^{\sigma}, \nu_{\frac{1}{2}}^{\sigma}\right)$ and $\mathrm{Cpl}_{\sigma}^{1,3}$ is the optimal coupling for $\left(\nu_{0}^{\sigma}, \nu_{1}^{\sigma}\right)$ and 
1) $\left(\nu_{t}^{\sigma}\right)_{t \in\left[0, \frac{1}{2}\right]}$ is a geodesic segment in Wasserstein space $\mathcal{W}_{2}\left(\bar{\Omega}, \mathrm{d}_{\mathrm{g}}\right)$;

2) Given $\delta \in\left(0, \frac{1}{2}\right),\left(\nu_{t}^{\sigma}\right)_{t \in\left[0, \frac{1}{2}-\delta\right] \cup\{1\}}$ have uniformly bounded $\mathfrak{m}$-densities;

3) $\mathfrak{m}\left(\operatorname{supp} \nu_{0}^{\sigma}\right) \gtrsim \sigma$ and $\mathfrak{m}\left(\operatorname{supp} \nu_{1}^{\sigma}\right) \gtrsim \sigma$ (by (3.48), (3.49) and (3.43)).

Moreover, since $\mathcal{H}^{n-1}(\partial \Omega \backslash R)=0$, by Rauch's comparison theorem we know $\operatorname{Vol}_{\mathrm{g}}\left(\operatorname{supp} \nu_{\frac{1}{2}}^{\sigma}\right) \lesssim \sigma^{n}$, so that $\mathfrak{m}\left(\operatorname{supp} \nu_{\frac{1}{2}}^{\sigma}\right) \lesssim \sigma^{n}$.

Since $\left(\bar{\Omega}, \mathrm{d}_{\Omega}, \mathfrak{m}\right)$ is $\operatorname{CD}(K, \infty)$, by Lemma $3.1[27]$ there exists a $L^{2}$-Wasserstein geodesic $\left(\bar{\nu}_{t}^{\sigma}\right)_{t \in[0,1]} \subset \mathcal{W}_{2}\left(\bar{\Omega}, \mathrm{d}_{\Omega}\right)$ with uniformly bounded densities, connecting $\nu_{\frac{1}{4}}^{\sigma}$ and $\nu_{1}^{\sigma}$ such that

$$
\mathfrak{m}\left(\operatorname{supp} \bar{\nu}_{t}^{\sigma}\right) \gtrsim \min \left\{\mathfrak{m}\left(\operatorname{supp} \nu_{\frac{1}{4}}^{\sigma}\right), \mathfrak{m}\left(\operatorname{supp} \nu_{1}^{\sigma}\right)\right\}, \quad t \in[0,1] .
$$

It is known that Riemannian manifolds are essentially non-branching, hence $\nu_{s}^{\sigma} \in$ $\left(\bar{\nu}_{t}^{\sigma}\right)$ for all $s \in\left[\frac{1}{4}, \frac{1}{2}\right]$. In particular, there exists $t_{1} \in(0,1)$ such that $\nu_{\frac{1}{2}}^{\sigma}=\bar{\nu}_{t_{1}}^{\sigma}$. Therefore we have

$$
\sigma^{n} \gtrsim \mathfrak{m}\left(\operatorname{supp} \nu_{\frac{1}{2}}^{\sigma}\right)=\mathfrak{m}\left(\operatorname{supp} \bar{\nu}_{t_{1}}^{\sigma}\right) \gtrsim O(\sigma)
$$

which is the contradiction. Therefore $\Pi^{\epsilon}\left(\Gamma^{3}\right)=0$.

In conclusion, we have proved that $\left(\bar{\Omega}, \mathrm{d}_{\Omega}, \mathfrak{m}\right)$ is $(M, \mathrm{~g})$-geodesically convex. By Lemma 3.2 we have $\left.\mathfrak{m}\right|_{\partial \Omega}=0$. Therefore $\mathfrak{m}=\left.e^{-V} \operatorname{Vol}_{\mathfrak{g}}\right|_{\Omega}$ for some Lipschitz function $V$. In particular, $\left(\bar{\Omega}, \mathrm{d}_{\Omega}, \mathfrak{m}\right)$ is infinitesimally Hilbertian and it satisfies $\operatorname{RCD}(K, \infty)$ condition.

\section{References}

[1] R. Alexander and S. Alexander, Geodesics in Riemannian manifoldswith-boundary, Indiana Univ. Math. J., 30 (1981), pp. 481-488.

[2] S. B. Alexander, I. D. Berg, And R. L. Bishop, The Riemannian obstacle problem, Illinois J. Math., 31 (1987), pp. 167-184.

[3] L. Ambrosio, Calculus, heat flow and curvature-dimension bounds in metric measure spaces, Proceedings of the ICM 2018, (2018).

[4] L. Ambrosio And N. Gigli, A user's guide to optimal transport. Modelling and Optimisation of Flows on Networks, Lecture Notes in Mathematics, Vol. 2062, Springer, 2011.

[5] L. Ambrosio, N. Gigli, A. Mondino, and T. Rajala, Riemannian Ricci curvature lower bounds in metric measure spaces with $\sigma$-finite measure, Trans. Amer. Math. Soc., 367 (2015), pp. 4661-4701. 
[6] L. Ambrosio, N. Gigli, And G. Savaré, Calculus and heat flow in metric measure spaces and applications to spaces with Ricci bounds from below, Invent. math., 195 (2014), pp. 289-391.

[7] —, Metric measure spaces with riemannian Ricci curvature bounded from below, Duke Math. J., 163 (2014), pp. 1405-1490.

[8] —, Bakry-Émery curvature-dimension condition and Riemannian Ricci curvature bounds, Ann. Probab., 43 (2015), pp. 339-404.

[9] F. Cavalletti, Decomposition of geodesics in the Wasserstein space and the globalization problem, Geom. Funct. Anal., 24 (2014), pp. 493-551.

[10] F. Cavalletti And A. Mondino, Measure rigidity of Ricci curvature lower bounds, Adv. Math., 286 (2016), pp. 430-480.

[11] — Sharp and rigid isoperimetric inequalities in metric-measure spaces with lower Ricci curvature bounds, Invent. Math., 208 (2017), pp. 803-849.

[12] J. CheEger, Differentiability of Lipschitz functions on metric measure spaces, Geom. Funct. Anal., 9 (1999), pp. 428-517.

[13] D. Cordero-Erausquin, R. J. McCann, and M. Schmuckenschläger, A Riemannian interpolation inequality à la Borell, Brascamp and Lieb, Invent. Math., 146 (2001), pp. 219-257.

[14] G. De Philippis And N. Gigli, Non-collapsed spaces with Ricci curvature bounded from below, J. Éc. polytech. Math., 5 (2018), pp. 613-650.

[15] M. Erbar And K.-T. Sturm, Rigidity of cones with bounded Ricci curvature, to appear in J. Eur. Math. Soc., (2017).

[16] N. GigLI, On the differential structure of metric measure spaces and applications, Mem. Amer. Math. Soc., 236 (2015), pp. vi+91.

[17] — Nonsmooth differential geometry - an approach tailored for spaces with Ricci curvature bounded from below, Mem. Amer. Math. Soc., 251 (2018), pp. $\mathrm{v}+161$.

[18] R. E. GReEne AND H. Wu, On the subharmonicity and plurisubharmonicity of geodesically convex functions, Indiana Univ. Math. J., 22 (1972/73), pp. 641653.

[19] B.-X. HAN, Ricci tensor on smooth metric measure space with boundary. Preprint, arXiv:1709.10143, 2017.

[20] — Measure rigidity of synthetic lower ricci curvature bound on riemannian manifolds. Preprint, arXiv:1902.00942, 2019.

[21] V. Kapovitch, M. Kell, And C. Ketterer, On the structure of RCD spaces with upper curvature bounds. Preprint, arXiv:1908.07036, 2019. 
[22] V. Kapovitch And C. KetTerer, Weakly noncollapsed RCD spaces with upper curvature bounds. Preprint, arXiv:1901.06966, 2019.

[23] M. KeLL, Transport maps, non-branching sets of geodesics and measure rigidity, Adv. Math., 320 (2017), pp. 520-573.

[24] B. Klartag, Needle decompositions in Riemannian geometry, Mem. Amer. Math. Soc., 249 (2017), pp. v +77.

[25] J. LotT And C. Villani, Ricci curvature for metric-measure spaces via optimal transport, Ann. of Math. (2), 169 (2009), pp. 903-991.

[26] B. O'NeILl, Semi-Riemannian geometry, vol. 103 of Pure and Applied Mathematics, Academic Press, Inc. [Harcourt Brace Jovanovich, Publishers], New York, 1983. With applications to relativity.

[27] T. Rajala, Local Poincaré inequalities from stable curvature conditions on metric spaces, Calc. Var. Partial Differential Equations, 44 (2012), pp. 477494.

[28] T. Rajala And K.-T. Sturm, Non-branching geodesics and optimal maps in strong $C D(K, \infty)$-spaces, Calc. Var. Partial Differential Equations, 50 (2014), pp. 831-846.

[29] G. SAVARÉ, Self-improvement of the Bakry-émery condition and Wasserstein contraction of the heat flow in $R C D(K, \infty)$ metric measure spaces, Disc. Cont. Dyn. Sist. A, 34 (2014), pp. 1641-1661.

[30] K.-T. Sturm, On the geometry of metric measure spaces. I, Acta Math., 196 (2006), pp. 65-131.

[31] _ - On the geometry of metric measure spaces. II, Acta Math., 196 (2006), pp. $133-177$.

[32] C. UdRIŞTE, Convex functions and optimization methods on Riemannian manifolds, vol. 297 of Mathematics and its Applications, Kluwer Academic Publishers Group, Dordrecht, 1994.

[33] M.-K. VOn Renesse And K.-T. Sturm, Transport inequalities, gradient estimates, entropy, and Ricci curvature, Comm. Pure Appl. Math., 58 (2005), pp. 923-940.

[34] F.-Y. WAng, Analysis for diffusion processes on Riemannian manifolds, vol. 18 of Advanced Series on Statistical Science \& Applied Probability, World Scientific Publishing Co. Pte. Ltd., Hackensack, NJ, 2014. 